\title{
HETEROCYCLES 47. SYNTHESIS, CHARACTERIZATION AND BIOLOGICAL EVALUATION OF SOME NEW THIAZOLE AURONES AS ANTIPROLIFERATIVE AGENTS
}

\author{
FANA-MARIA COMAN ${ }^{1}$, ARMELLE T. MBAVENG ${ }^{2,3}$, GABRIEL MARC $^{4}$, DENISA LEONTE $^{1}$, \\ BALÁZS BRÉM $^{5}$, LAURIAN VLASE ${ }^{6}$, SILVIA IMRE ${ }^{7}$, VICTOR KUETE ${ }^{2,3}$, VALENTIN \\ ZAHARIA $^{1 *}$ \\ 1 "Iuliu Haţieganu” University of Medicine and Pharmacy, Faculty of Pharmacy, Department of Organic Chemistry, 41 \\ Victor Babes Street, 400012, Cluj-Napoca, Romania \\ 2 “Johannes Gutenberg” University, Institute of Pharmacy and Biochemistry, Department of Pharmaceutical Biology, \\ Stawdenger Weg 5, 55128, Mainz, Germany \\ ${ }^{3}$ University of Dschang, Faculty of Science, Department of Biochemistry, Cameroon \\ 4 "Iuliu Hatieganu" University of Medicine and Pharmacy, Department of Pharmaceutical Chemistry, 8 Victor Babeș Street, \\ 400012, Cluj-Napoca, Romania \\ 5 "Babeş-Bolyai" University, Faculty of Chemistry and Chemical Engineering, 11 Arany János Street, 400028, Cluj-Napoca, \\ Romania \\ 6 "Iuliu Haţieganu” University of Medicine and Pharmacy, Department of Pharmaceutical Technology and Biopharmacy, 41 \\ Victor Babeș Street, 400012, Cluj-Napoca, Romania \\ ${ }^{7}$ University of Medicine, Pharmacy, Science and Technology of Târgu Mureș, Department of Analytical Chemistry and Drug \\ Analysis, 38 Gheorghe Marinescu Street, 540139, Târgu Mureș, Romania
}

*corresponding author: vzaharia@umfcluj.ro

Manuscript received: November 2019

\begin{abstract}
New substituted thiazole aurones 2a-o were synthesized and evaluated for their anticancer activity. A screening of methods based on the oxidative cyclization of ortho-hydroxychalcones, with different agents, was applied in order to find the optimal way for the synthesis of these compounds. The best oxidizing agent proved to be mercury(II) acetate and it allowed the synthesis of the thiazole aurones with yields of $70-86 \%$. All synthesized compounds were purified and characterized by ESI-MS, ${ }^{1} \mathrm{H}$ NMR, ${ }^{13} \mathrm{C}$ NMR and IR. The cytotoxicity of the thiazole aurones was determined in a panel of nine cancer cell lines using doxorubicin as control. Compounds $\mathbf{2 a}, \mathbf{2} \mathbf{i}$ and $\mathbf{2 e}$ displayed the best cytotoxic activities. The interactions between aurones 2a-o and topoisomerases I and II were assessed by means of the molecular docking study and their target molecule is predicted to be topoisomerase I. The evaluation of the results revealed the importance of the thiazole ring for establishing a hydrogen bond with His367 and Arg364.
\end{abstract}

\section{Rezumat}

O serie de noi aurone tiazolice $\mathbf{2 a - 0}$ au fost sintetizate, caracterizate și evaluate pentru potențialul antiproliferativ. Au fost aplicate diverse metode de ciclizare oxidativă a orto-hidroxicalconelor tiazolice, cu diverși agenți oxidanți, și s-a observat faptul că agentul oxidant cel mai eficient pentru obținerea auronelor tiazolice a fost acetatul de mercur(II). Noile aurone tiazolice s-au obținut cu randamente cuprinse între $70 \%$ și $86 \%$. Toți compuşii sintetizați au fost purificaţi și caracterizați prin metode spectrale (IR, ${ }^{1} \mathrm{H}$ RMN, ${ }^{13} \mathrm{C}$ RMN şi SM). Citotoxicitatea auronelor tiazolice a fost evaluată pe nouă linii celulare canceroase utilizând doxorubicina ca substanță antiproliferativă de referință. Compușii 2a, 2i și 2e au prezentat cele mai bune efecte citotoxice. Studiul interacțiunilor dintre compușii sintetizați și topoizomerazele I și II sugerează faptul că molecula țintă a auronelor este topoizomeraza I, precum și importanța tiazolului în stabilirea unor legături de hidrogen cu His367 și cu Arg364 din această enzimă.

Keywords: thiazole aurone, antiproliferative activity, topoisomerase

\section{Introduction}

Cancer is one of the main causes of death worldwide and its frequency increases year by year. Cancer affects many types of cells, leading to various and multiple forms of tumours which can develop in both children and adults. Most drugs used in the cancer therapy nowadays are not selective and they determine the death of healthy and cancerous cells alike $[13,21$, 37]. Therefore, new drugs with higher selectivity for cancerous cells and, consequentially, fewer side effects are still needed.

Many naturally occurring compounds with different structures have showed anticancer activity. In the past years, flavonoids have been found to be a group of potential anticancer agents $[37,46]$. Evidence suggests 
that one of the anticancer effects displayed by some flavonoids is based on the inhibition of topoisomerases. Some of the discovered and tested flavonoids were proved to be topoisomerase I inhibitors, whereas others were proved to inhibit both types of topoisomerases, acting by stabilizing in vitro the topoisomerase-DNA complexes. Topoisomerases are essential enzymes that play a pivotal role in the over-winding or under-winding of the DNA, caused by the intertwined nature of its double-helical structure [27, 29, 30].

Aurones, compounds from the family of flavonoids, contribute to the bright yellow colour of some flowering plants such as cosmos, snapdragon and dahlia. Among the various subclasses of flavonoids, aurones have not been extensively studied for their biological activities
$[3,6,10,13,21,37]$. However, the existing data from the literature that refers to the anticancer activity of aurones and their synthetic analogues is very promising [7, 10, 13, 20, 21, 26, 37]. Alsayari et al. showed that aurones exhibit a broad spectrum of anticancer mechanisms by interacting with various targets, such as cyclin dependent kinase, histone deacetylase, topoisomerase, adenosine receptor or telomerase [2]. Aurones are compounds that contain a benzofuranone ring linked to a benzene ring by a vinylic carbon bridge $[13,21,37]$. This suggests that there are two sites on the aurone core, where modifications can be made, in order to obtain substituted analogues with enhanced anticancer activity (Figure 1).

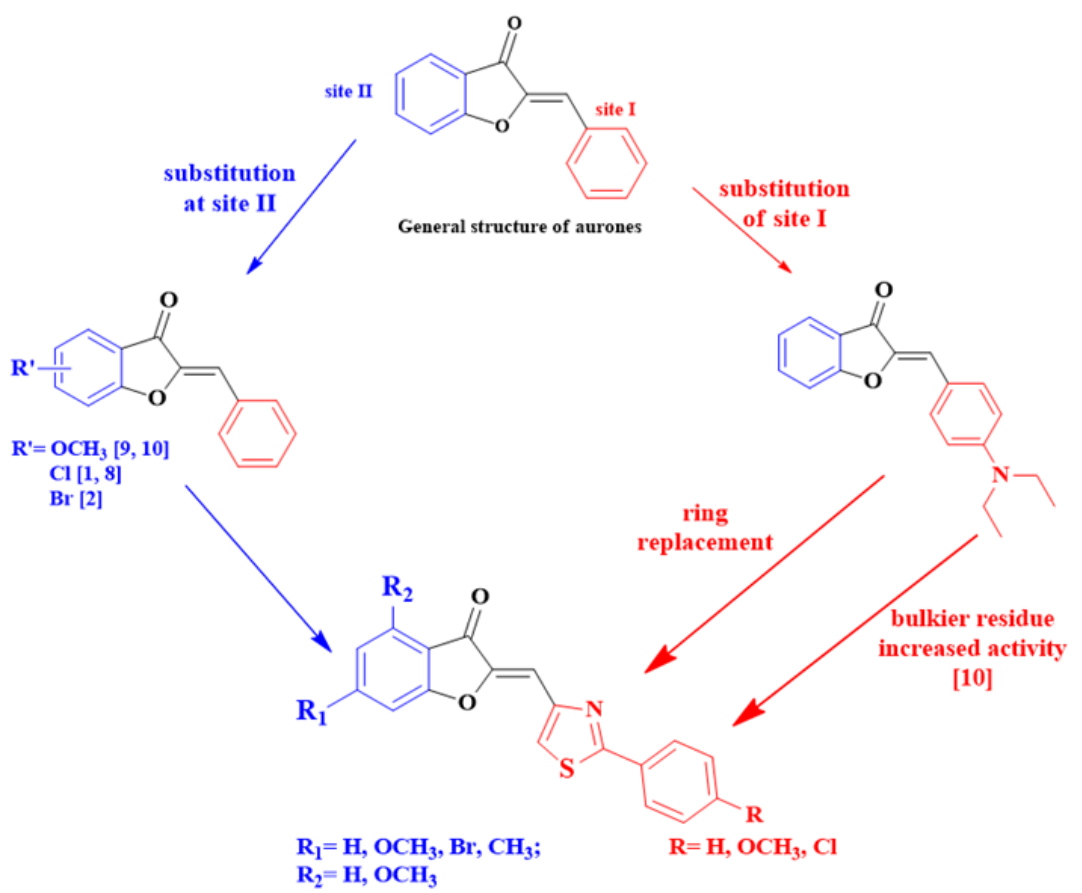

Figure 1.

The design strategy for the target thiazole aurones

The biological results obtained by Cheng et al. suggest that the presence of a moiety that contains a nitrogen atom enhanced the anticancer activity of the tested compounds. Moreover, the presence of a bulkier substituent close to this nitrogen atom increased the activity. A deeper analysis revealed that the nitrogen atom should not be part of a strong electron withdrawing group, such as an amide or a nitrile [7].

Based on these findings, we proposed the synthesis of a new series of aurones, substituted on site I, with a scaffold where the nitrogen atom is part of a $\pi$ excessive heterocyclic ring, without bonding the nitrogen atom with a bulkier group, so the nitrogen will not be deprived of electrons and it will not lose the ability to form a hydrogen bond with the Arg364 or His367 from the active site of topoisomerase I, as suggested by the molecular docking studies.
We guided our research towards the thiazole ring, because previous studies reported in literature stated that the benzene ring bound to the benzofuranone through the vinylic carbon is not mandatory and it can be replaced with other cyclic compounds containing nitrogen, some of these compounds exhibiting remarkable activity $[10,44]$. Thiazole is already a common moiety found in the structure of certain molecules with anticancer activity $[1,14,16,19,42]$, thus the research concerning the biological properties of the thiazole ring is ongoing and warrants further investigation. The hydrocarbonic substitution of the thiazole in position 2 was realized with a phenyl ring which, in some compounds from our series, was substituted in para position.

Among the reported aurones with anticancer activity, two types of substitution can be found on site II, in 
terms of volume of the substituent linked directly to the benzofuranone. Here, substitutions can be made on one of the four available positions from the benzene fragment of benzofuranone. Most of the compounds reported in literature are substituted with small residues, such as chlorine, methoxy, hydroxyl or amino groups $[7,10,13,26]$ or, less often, with bulky substituents containing various aromatic rings [5]. We have chosen small substituents, inspired by the idea that insertion of the phenylthiazole system on substitution site I, already extended the molecules in our series and we wanted to avoid obtaining molecules with a higher molecular weight which can decrease the permeability through cell membranes [28].

Since the anticancer activity of thiazole aurones has not been extensively studied, this investigation deals with the synthesis, evaluation of the antiproliferative activity and assessment of the theoretical interactions of some new synthetic aurones analogues with the human topoisomerases I and II.

\section{Materials and Methods}

\section{Chemistry}

All used chemicals, solvents and reagents, were of 95 - 99\% purity grade and purchased from Alfa Aesar and Sigma Aldrich, Germany. Normal and deuterated solvents were used as received without further purifications.

The reactions were monitored by performing TLC (silica gel, aluminium sheets $60 \mathrm{~F}_{254}$, Merck) using dichloromethane, dichloromethane:acetone $=25: 1$ $(\mathrm{v} / \mathrm{v})$ or dichloromethane:acetone $=9: 1(\mathrm{v} / \mathrm{v})$ as mobile phases. The TLC plates were analysed using a UV lamp, at $254 \mathrm{~nm}$ or $365 \mathrm{~nm}$ wavelengths.

The compounds were purified by column chromatography, using different solvents mixtures (dichloromethane, dichloromethane:acetone $=9: 1(\mathrm{v} / \mathrm{v})$ or dichloromethane:acetone $=25: 1(\mathrm{v} / \mathrm{v}))$ as mobile phases, as indicated for each individual compound, in the experimental part.

Purity of compounds was checked preliminary by TLC and then using RP-HPLC coupled with MS spectrometry (Agilent 1100 series) in order to confirm that the purification of the target compounds was successfully achieved.

Mass spectra were recorded on an Agilent 1100 Ion Trapp SL mass spectrometer (Agilent, Santa Clara, CA, USA), operated in positive ionization mode detection, using an electrospray ionization source at $70 \mathrm{eV}$.

Melting points were determined in an open glass capillary tube on an Electrothermal 9000 IA digital apparatus.

The ${ }^{1} \mathrm{H}$ NMR, ${ }^{13} \mathrm{C}$ NMR, ${ }^{1} \mathrm{H}^{-1} \mathrm{H}$ COSY and ${ }^{1} \mathrm{H}^{-13} \mathrm{C}$ HMQC spectra were recorded on a Bruker (Ascend, BioSpin, Germany) Avance spectrometer operating at $600 \mathrm{MHz}$ and $151 \mathrm{MHz}$, respectively, in different deuterated solvents (chloroform-d, dimethylsulfoxide$\mathrm{d}_{6}$ ); the chemical shifts are expressed in $\delta \mathrm{ppm}$.

The FT-IR analysis was performed on a 460 Plus spectrometer (Jasco) at the University of Medicine, Pharmacy, Science and Technology of Târgu Mureș, Romania, Department of Analytical Chemistry and Drug Analysis by using the Spectra Manager software. The solid sample was introduced in the ATR device's slot and the IR spectra were recorded between $4000 \mathrm{~cm}^{-1}$ and $400 \mathrm{~cm}^{-1}$ wavelengths at $4 \mathrm{~cm}^{-1}$ resolution.

General synthetic procedures

The cyclization of thiazole ortho-hydroxychalcone la with copper(II) acetate in DMSO

2-Phenylthiazole ortho-hydroxychalcone $1 a$ (0.91 mmol, $0.28 \mathrm{~g}$ ) was added to a stirred solution of copper(II) acetate $(0.655 \mathrm{mmol}, 0.13 \mathrm{~g})$ in DMSO $(2.8 \mathrm{~mL})$ at room temperature and refluxed for $12 \mathrm{~h}$ on an electric mantle. The reaction was monitored by TLC, using dichloromethane:acetone $=25: 1(v / v)$ as the mobile phase. This showed that an unexpected product was formed in an approximate molar ratio of $1: 1$, alongside the aurone $\mathbf{2 a}$. We identified the product as the corresponding flavone $\mathbf{3 a}$, based on spectral data.

2-(2-Phenylthiazol-4-yl)chroman-4-one (3a): mp: $189-190^{\circ} \mathrm{C}$; ${ }^{1} \mathrm{H}$ NMR (600 MHz, $\mathrm{CDCl}_{3}$ )s $\delta 8.24$ (dd, $J=7.9,1.2 \mathrm{~Hz}, 1 \mathrm{H}, \mathrm{CH}-5$ chromen-4-one), 8.04 (s, $1 \mathrm{H}, \mathrm{CH}-5$ thiazole), $8.01(\mathrm{dd}, J=6.5,2.9 \mathrm{~Hz}, 2 \mathrm{H}$, CH-2', CH-6'), 7.72 - 7.69 (m, 1H, CH-7), 7.55 (d, $J=8.3 \mathrm{~Hz}, 1 \mathrm{H}, \mathrm{CH}-8$ chromen-4-one), $7.50-7.47$ (m, 3H, CH-3', CH-4', CH-5'), 7.42 (t, $J=7.5 \mathrm{~Hz}, 1 \mathrm{H}$, CH-6 chromen-4-one), 7.39 (s, 1H, CH-3 chromen4-one); ${ }^{13} \mathrm{C}$ NMR (151 MHz, $\left.\mathrm{CDCl}_{3}\right) \delta 178.82(\mathrm{C}$, $\mathrm{C}=\mathrm{O}), 169.42$ (C, C-2 thiazole), 158.71 (C, C-9 chromen4-one), 156.21 (C, C-2 chromen-4-one), 148.74 (C, C-4 thiazole), 134.09 (C, C-1'), 132.83 (CH, C-5 thiazole), 131.03 (CH, C-7 chromen-4-one), 129.26 (CH, C-3', C-5'), 126.91 (CH, C-2', C-6'), 126.01 (CH, C-4'), 125.48 (CH, C-5 chromen-4-one), 124.25 (CH, C-6 chromen-4-one), 120.41 (C, C-10 chromen4-one), 118.11 ( $\mathrm{CH}, \mathrm{C}-8$ chromen-4-one), 108.64 (CH, C-3 chromen-4-one). MS: $m / z 308.30\left[\mathrm{M}+\mathrm{H}^{+}\right]$ (calculated for $\mathrm{C}_{18} \mathrm{H}_{14} \mathrm{NO}_{2} \mathrm{~S}^{+}: 308.07$ ).

The cyclization of thiazole ortho-hydroxychalcone $\mathbf{1 a}$ with hydrogen peroxide in alkaline environment

A solution of $30 \%$ hydrogen peroxide $(0.42 \mathrm{~mL})$ was added to a mixture of 2-phenylthiazole ortho-hydroxychalcone 1a (0.6 mmol, $0.19 \mathrm{~g})$, ethanol $(1.8 \mathrm{~mL})$ and a $20 \%$ aqueous solution of sodium hydroxide (1.8 $\mathrm{mL}$ ) with continuous stirring at room temperature for $48 \mathrm{~h}$. The reaction mixture was acidified with acetic acid and the solid that was formed was filtered, washed with water and purified by column chromatography. The reaction was monitored by TLC, using dichloromethane:acetone $=25: 1(\mathrm{v} / \mathrm{v})$ as the mobile phase. The structural analysis revealed the formation of an 
FARMACIA, 2020, Vol. 68, 3

unexpected product, the corresponding hydroxyflavone 4a as the main product.

3-Hydroxy-2-(2-phenylthiazol-4-yl)-4H-chromen-4one (4a): mp: $203-204^{\circ} \mathrm{C} ;{ }^{1} \mathrm{H}$ NMR $(600 \mathrm{MHz}$, DMSO) $\delta 10.17$ (s, 1H, hydroxyl), 8.55 (s, 1H, CH-5 thiazole), 8.13 (d, 1H, CH-5 chromen-4-one), 8.07 (d, 2H, CH-2', CH-6'), 7.82 (t, 1H, CH-6 chromen4-one), 7.76 (d, 1H, CH-8 chromen-4-one), 7.57 (m, 3H, CH-3', CH-4', CH-5'), 7.49 (t, 1H, CH-7 chromen4-one). ${ }^{13} \mathrm{C}$ NMR (151 MHz, DMSO) $\delta 172.99$ (C, $\mathrm{C}=\mathrm{O}$ ), 167.77 (C, C-2 thiazole), 154.81 (C, C-8a chromen4-one), 146.91 (C, C-4 thiazole), 141.89 (CH, C-3 chromen-4-one), 139.74 (C, C-1'), 134.36 (CH, C-7 chromen-4-one), 132.77 (CH, C-2 chromen-4-one), 131.37 (CH, C-4'), 129.90 (CH, C-3', C-5'), 126.97 (CH, C-2', C-6'), 125.37 (CH, C-5 thiazole), 125.12 (CH, C-5 chromen-4-one), 124.01 (CH, C-6 chromen4-one), 122.39 (C, C-4a chromen-4-one), 118.95 (CH, C-8 chromen-4-one). ESI ${ }^{+}$MS: $m / z 322.00$ (calculated for $\mathrm{C}_{18} \mathrm{H}_{12} \mathrm{NO}_{3} \mathrm{~S} 322.05[\mathrm{M}+\mathrm{H}]^{+}$).

The cyclization of thiazole ortho-hydroxychalcone $\mathbf{1 a}$ with selenium dioxide

A mixture of freshly sublimed selenium dioxide (1.98 mmol) and 2-phenylthiazole ortho-hydroxychalcone $1 \mathrm{a}(0.75 \mathrm{mmol}, 0.23 \mathrm{~g})$ dissolved in n-butanol (3.30 $\mathrm{mL}$ ) was heated under reflux for $24 \mathrm{~h}$. The obtained turbid solution was filtered while hot to remove the selenium metal. The reaction was monitored by TLC, using dichloromethane:acetone $=25: 1(v / v)$ as the mobile phase and this showed that the obtained products were not aurones, but other products from the flavonoid family, which we later identified as the corresponding flavone 3a and the corresponding hydroxyflavone $\mathbf{4 a}$, based on spectral data.

The cyclization of thiazole ortho-hydroxychalcone 1a-o with mercury(II) acetate in pyridine

The thiazole ortho-hydroxychalcone 1a-o (1 mmol) was mixed with mercury(II) acetate $(1.1 \mathrm{mmol})$ in 10 - $20 \mathrm{~mL}$ pyridine at room temperature. The mixture was refluxed and stirred at $110^{\circ} \mathrm{C}$ for $10-20 \mathrm{~h}$ in an oil bath. The cooled reaction mixture was poured into ice cold water and acidified with $\mathrm{HCl} \mathrm{10 \% .} \mathrm{The}$ formed yellow precipitate was filtered and purified by column chromatography with different solvents mixtures as mobile phases, as indicated for each individual compound.

(Z)-2-((2-phenylthiazol-4-yl)methylene)benzofuran$3(2 H)$-one $(2 a)$ : bright-yellow powder, purified by column chromatography (eluent: dichlorometane:acetone = 25:1), Yield $=80 \% ; \mathrm{mp}: 169.1-170.8^{\circ} \mathrm{C} ; R_{\mathrm{f}}=0.5$ (eluent: dichloromethane: acetone $=25: 1 \mathrm{v} / v)$. IR: $v\left(\mathrm{~cm}^{-1}\right)$ : 3093.26 (C-H aromatic), 1593.88 (C=O). ${ }^{1} \mathrm{H}$ NMR $\left(600 \mathrm{MHz}, \mathrm{CDCl}_{3}\right) \delta 8.16(\mathrm{~s}, 1 \mathrm{H}, \mathrm{CH}-5$ thiazole), 8.02 - 8.00 (m, 2H, CH-2', CH-6'), 7.82 (d, 1H, CH-4, $J=7.6 \mathrm{~Hz}$ ), 7.68 (t, 1H, CH-6), 7.48 (m, 3H, CH-3', CH-4', CH-5'), 7.34 (d, 1H, CH-7, J = 8.2 Hz ), 7.25 (m, 2H, thiazole- $\mathrm{CH}=\mathrm{C}<, \mathrm{CH}-5) .{ }^{13} \mathrm{C}$ NMR $(151 \mathrm{MHz}$, $\left.\mathrm{CDCl}_{3}\right) \delta 184.3(\mathrm{C}, \mathrm{C}=\mathrm{O}), 167.8(\mathrm{C}, \mathrm{C}-2$ thiazole $)$,
166.0 (C, C-8), 149.4 (C, C-2), 147.6 (C, C-4 thiazole), 136.9 (CH, C-6), 133.0 (C, C-1'), 130.5 (CH, C-4'), 129.0 (CH, C-3', C-5'), 126.8 (CH, C-2', C-6'), 124.8 (CH, C-4), 123.8 (CH, C-5 thiazole), 123.7 (CH, C-5), 121.9 (C, C-9), 112.9 (CH, C-7), 106.4 (CH, thiazole$\mathbf{C H}=\mathrm{C}<$ ). $\mathrm{ESI}^{+}-\mathrm{MS}: m / z 306.30$ (calculated for $\mathrm{C}_{18} \mathrm{H}_{12} \mathrm{NO}_{2} \mathrm{~S}$ $\left.306.05[\mathrm{M}+\mathrm{H}]^{+}\right)$.

(Z)-2-((2-(4-methoxyphenyl)thiazol-4-yl)methylene)benzofuran-3(2H)-one $(\mathbf{2 b})$ : yellow powder, purified by column chromatography (eluent: dichlorometane: acetone $=25: 1)$, Yield $=76 \% ; \mathrm{mp}: 212.4-213.7^{\circ} \mathrm{C}$; $R_{\mathrm{f}}=0.5$ (eluent: dichloromethane:acetone $=25: 1 \mathrm{v} / \mathrm{v}$ ). IR: $v\left(\mathrm{~cm}^{-1}\right)$ : $3093.26(\mathrm{C}-\mathrm{H}$ aromatic $), 1597.73(\mathrm{C}=\mathrm{O})$. ${ }^{1} \mathrm{H}$ NMR $\left(600 \mathrm{MHz}, \mathrm{CDCl}_{3}\right) \delta 8.11(\mathrm{~s}, 1 \mathrm{H}, \mathrm{CH}-5$ thiazole), 7.96 (d, 2H, CH-2', CH-6', $J=8.9 \mathrm{~Hz}$ ), 7.84 (d, 1H, CH-4, J = 7.7 Hz), 7.69 (t, 1H, CH-6), 7.35 (d, 1H, CH-7, J = 8.4 Hz), 7.27-7.24 (m, 2H, thiazole- $\mathrm{CH}=\mathrm{C}<, \mathrm{CH}-5), 7.01$ (d, 2H, CH-3', CH-5', $J=8.9 \mathrm{~Hz}), 3.89$ (s, 3H, OCH 3$).{ }^{13} \mathrm{C} \mathrm{NMR}(151 \mathrm{MHz}$, $\left.\mathrm{CDCl}_{3}\right) \delta 184.3(\mathrm{C}, \mathrm{C}=\mathrm{O}), 167.6(\mathrm{C}, \mathrm{C}-2$ thiazole $)$, 165.9 (C, C-8), 161.5 (C, C-2), 149.1 (C, C-4'), 147.5 (C, C-4 thiazole), 136.9 (CH, C-6), $128.3(\mathrm{CH}, \mathrm{C}-2$ ', C-6'), 125.9 (C, C-1'), 124.7 (CH, C-4), 123.6 (CH, C-5), 123.1 (CH, C-5 thiazole), 121.9 (C, C-9), 114.4 (CH, CH-3', CH-5'), 112.9 (CH, C-7), 106.5 (CH,

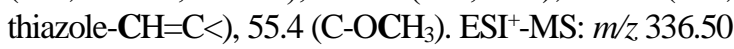
(calculated for $\mathrm{C}_{19} \mathrm{H}_{14} \mathrm{NO}_{3} \mathrm{~S} 336.06[\mathrm{M}+\mathrm{H}]^{+}$).

(Z)-2-((2-(4-chlorophenyl)thiazol-4-yl)methylene)benzofuran-3(2H)-one $(\mathbf{2 c})$ : yellow powder, purified by column chromatography (eluent: dichlorometane: acetone $=25: 1)$, Yield $=78 \%$; $\mathrm{mp}: 197.1-198.0^{\circ} \mathrm{C}$; $R_{\mathrm{f}}=0.5$ (eluent: dichloromethane:acetone $=25: 1 \mathrm{v} / \mathrm{v}$ ). IR: $v\left(\mathrm{~cm}^{-1}\right)$ : 3046.98 (C-H aromatic), $1599.66(\mathrm{C}=\mathrm{O})$. ${ }^{1} \mathrm{H}$ NMR $\left(600 \mathrm{MHz}, \mathrm{CDCl}_{3}\right) \delta 8.14(\mathrm{~s}, 1 \mathrm{H}, \mathrm{CH}-5$ thiazole), 7.92 (d, 2H, CH-2', CH-6', $J=8.2 \mathrm{~Hz}$ ), 7.81 (d, 1H, CH-4, J = 7.6 Hz), 7.67 (t, 1H, CH-6), 7.44 (d, 2H, CH-3', CH-5', J = 8.2 Hz), 7.32 (d, $1 \mathrm{H}, \mathrm{CH}-7, J=8.3 \mathrm{~Hz}$ ), 7.24 (t, 1H, CH-5), 7.20 (s, $1 \mathrm{H}$, thiazole- $\mathrm{CH}=\mathrm{C}<) .{ }^{13} \mathrm{C}$ NMR $\left(151 \mathrm{MHz}, \mathrm{CDCl}_{3}\right)$ $\delta 184.2(\mathrm{C}, \mathrm{C}=\mathrm{O}), 166.3(\mathrm{C}, \mathrm{C}-2$ thiazole $), 165.9(\mathrm{C}$, C-8), 149.5 (C, C-2), 147.6 (C, C-4 thiazole), 137.0 (CH, C-6), 136.5 (C, C-1'), 131.5 (C, C-4'), 129.3 (CH, C-3', C-5'), 127.9 (CH, C-2', C-6'), 124.7 (CH, C-4), 123.9 (CH, C-5 thiazole), 123.7 (CH, C-5), 121.8 (C, C-9), $112.9(\mathrm{CH}, \mathrm{C}-7), 106.0(\mathrm{CH}$, thiazole$\mathbf{C H}=\mathrm{C}<)$. ESI ${ }^{+}-\mathrm{MS}: \mathrm{m} / z 340.7\left([\mathrm{M}+\mathrm{H}]^{+},{ }^{35} \mathrm{Cl}\right), 342.1$ $\left([\mathrm{M}+\mathrm{H}]^{+},{ }^{37} \mathrm{Cl}\right)\left(\right.$ calculated for $\mathrm{C}_{18} \mathrm{H}_{11} \mathrm{ClNO}_{2} \mathrm{~S} 340.01$ $\left.[\mathrm{M}+\mathrm{H}]^{+},{ }^{35} \mathrm{Cl}, 342.00[\mathrm{M}+\mathrm{H}]^{+},{ }^{37} \mathrm{Cl}\right)$.

(Z)-6-methoxy-2-((2-phenylthiazol-4-yl)methylene)benzofuran-3(2H)-one (2d): pale-yellow powder, purified by column chromatography (eluent: dichlorometane: acetone $=50: 1)$, Yield $=85 \%$; $\mathrm{mp}: 190.4$ $191.3^{\circ} \mathrm{C} ; R_{\mathrm{f}}=0.5$ (eluent: dichloromethane:acetone $=$ 50:1 v/v). IR: $v\left(\mathrm{~cm}^{-1}\right): 3107.72$ (C-H aromatic), 1604.48 $(\mathrm{C}=\mathrm{O}) .{ }^{1} \mathrm{H}$ NMR $\left(600 \mathrm{MHz}, \mathrm{CDCl}_{3}\right) \delta 8.09(\mathrm{~s}, 1 \mathrm{H}$, CH-5 thiazole), 8.02 - 8.00 (m, 2H, CH-2', CH-6'), $7.72(\mathrm{~d}, 1 \mathrm{H}, \mathrm{CH}-4, J=8.2 \mathrm{~Hz}), 7.49-7.47(\mathrm{~m}, 3 \mathrm{H}$, CH-3', CH-4', CH-5'), 7.18 (s, 1H, CH-7), 6.79 - 6.77 
FARMACIA, 2020, Vol. 68, 3

(m, 2H, CH-5, thiazole- $\mathrm{CH}=\mathrm{C}<$ ), $3.95\left(\mathrm{~s}, 3 \mathrm{H}, \mathrm{OCH}_{3}\right)$. ${ }^{13} \mathrm{C} \mathrm{NMR}\left(151 \mathrm{MHz}, \mathrm{CDCl}_{3}\right) \delta 182.5(\mathrm{C}, \mathrm{C}=\mathrm{O}), 168.4$ (C, C-2 thiazole), 167.7 (C, C-8), 167.5 (C, C-6), 149.5 (C, C-2), 148.5 (C, C-4 thiazole), 133.0 (C, C-1'), 130.5 (CH, C-4'), 129.0 (CH, C-3', C-5'), 126.7 (CH, C-2', C-6'), 125.9 (CH, C-4), $123.1(\mathrm{CH}$, C-5 thiazole), 115.0 (C, C-9), 112.2 (CH, C-5), 105.3 $(\mathrm{CH}, \mathrm{C}-7), 96.8(\mathrm{CH}$, thiazole- $\mathrm{CH}=\mathrm{C}<), 56.0\left(\mathrm{C}-\mathrm{OCH}_{3}\right)$. ESI ${ }^{+}$-MS: $m / z 336.2$ (calculated for $\mathrm{C}_{19} \mathrm{H}_{13} \mathrm{NO}_{3} \mathrm{~S} 336.07$ $\left.[\mathrm{M}+\mathrm{H}]^{+}\right)$.

(Z)-6-methoxy-2-((2-(4-methoxyphenyl)thiazol-4-yl)methylene)benzofuran-3(2H)-one (2e): pale-orange powder, purified by column chromatography (eluent: dichlorometane: acetone $=25: 1$ ), Yield $=70 \%$; $\mathrm{mp}$ : $192.0-193.4^{\circ} \mathrm{C} ; R_{\mathrm{f}}=0.5$ (eluent: dichloromethane: acetone $=25: 1 \mathrm{v} / \mathrm{v})$. IR: $v\left(\mathrm{~cm}^{-1}\right): 3122.19(\mathrm{C}-\mathrm{H}$ aromatic $)$, $1604.48(\mathrm{C}=\mathrm{O}) .{ }^{1} \mathrm{H}$ NMR $\left(600 \mathrm{MHz}, \mathrm{CDCl}_{3}\right) \delta 8.00$ (s, 1H, CH-5 thiazole), 7.93 (d, 2H, CH-2', CH-6', $J=8.6 \mathrm{~Hz}), 7.70(\mathrm{~d}, 1 \mathrm{H}, \mathrm{CH}-4, J=8.4 \mathrm{~Hz}), 7.13$ (s, 1H, CH-7), 6.98 (d, 2H, CH-3', CH-5', $J=8.6 \mathrm{~Hz}$ ), $6.76-6.75(\mathrm{~m}, 2 \mathrm{H}, \mathrm{CH}-5$, thiazole-CH=C<), 3.94 (s, 3H, OCH $3-\mathrm{C}-6), 3.88$ (s, 3H, OCH $3-\mathrm{C}-4$ ') ${ }^{13} \mathrm{C}$ NMR $\left(151 \mathrm{MHz}, \mathrm{CDCl}_{3}\right) \delta 182.5(\mathrm{C}, \mathrm{C}=\mathrm{O}), 168.4$ (C, C-2 thiazole), 167.5 (C, C-8), 167.4 (C, C-6) 161.4 (C, C-2), 149.2 (C, C-4'), 148.4 (C, C-4 thiazole), 128.2 (CH, C-2', C-6'), 126.0 (C, C-1'), 125.8 (CH, C-4), 122.4 (CH, C-5 thiazole), 115.1 (C, C-9), 114.3 (CH, C-3', C-5'), 112.1 (CH, C-5), 105.4 (CH, C-7), $96.8(\mathrm{CH}$, thiazole- $\mathrm{CH}=\mathrm{C}<), 56.06\left(\mathrm{OCH}_{3}-\mathrm{C}-6\right), 55.43$ $\left(\mathrm{OCH}_{3}-\mathrm{C}-4\right.$ '). ESI ${ }^{+} \mathrm{MS}: \mathrm{m} / z 366.1$ (calculated for $\left.\mathrm{C}_{20} \mathrm{H}_{16} \mathrm{NO}_{4} \mathrm{~S} 366.08[\mathrm{M}+\mathrm{H}]^{+}\right)$.

(Z)-2-((2-(4-chlorophenyl)thiazol-4-yl)methylene)6-methoxybenzofuran-3(2H)-one $(2 \mathrm{f})$ : pale-yellow powder, purified by column chromatography (eluent: dichlorometane: acetone $=50: 1)$, Yield $=70 \%$; $\mathrm{mp}$ : $212.6-213.5^{\circ} \mathrm{C} ; R_{\mathrm{f}}=0.5$ (eluent: dichloromethane: acetone $=50: 1 \mathrm{v} / v)$. IR: $v\left(\mathrm{~cm}^{-1}\right): 3113.51$ (C-H aromatic), $1608.34(\mathrm{C}=\mathrm{O}) ;{ }^{1} \mathrm{H} \mathrm{NMR}\left(600 \mathrm{MHz}, \mathrm{CDCl}_{3}\right) \delta 8.07$ (s, 1H, CH-5 thiazole), 7.93 (d, 2H, CH-2', CH-6', $J=8.5 \mathrm{~Hz}), 7.71(\mathrm{~d}, 1 \mathrm{H}, \mathrm{CH}-4, J=9 \mathrm{~Hz}), 7.44$ (d, $2 \mathrm{H}, \mathrm{CH}-2$ ', CH-5', $J=8.5 \mathrm{~Hz}$ ), 7.12 (s, 1H, CH-7), $6.77-6.76(\mathrm{~m}, 2 \mathrm{H}, \mathrm{CH}-5$, thiazole-CH=C<), 3.95 $\left(\mathrm{s}, 3 \mathrm{H}, \mathrm{OCH}_{3}\right) .{ }^{13} \mathrm{C} \mathrm{NMR}\left(151 \mathrm{MHz}, \mathrm{CDCl}_{3}\right) \delta 182.5$ $(\mathrm{C}, \mathrm{C}=\mathrm{O}), 168.4$ (C, C-2 thiazole), 167.5 (C, C-8), 166.3 (C, C-6), 149.7 (C, C-4 thiazole), 148.6 (C, C-2), 136.4 (C, C-1'), 131.6 (C, C-4'), 129.3 (CH, C-3', C-5'), 127.9 (CH, C-2', C-6'), $125.9(\mathrm{CH}, \mathrm{C}-4), 123.2(\mathrm{CH}$, C-5 thiazole), 115.0 (C, C-9), 112.2 (CH, C-5), 105.0 $(\mathrm{CH}, \mathrm{C}-7), 96.8(\mathrm{CH}$, thiazole- $\mathrm{CH}=\mathrm{C}<), 56.1(\mathrm{C}-$ $\left.\left.\mathrm{OCH}_{3}\right) . \mathrm{ESI}^{+}-\mathrm{MS}: \mathrm{m} / z, 370.1([\mathrm{M}+\mathrm{H}]]^{+}{ }^{35} \mathrm{Cl}\right), 372.1$ $\left.([\mathrm{M}+\mathrm{H}]]^{+}{ }^{37} \mathrm{Cl}\right)$ (calculated for $\mathrm{C}_{19} \mathrm{H}_{13} \mathrm{ClNO}_{3} \mathrm{~S} 370.03$ $\left.[\mathrm{M}+\mathrm{H}]^{+},{ }^{35} \mathrm{Cl}, 372.03[\mathrm{M}+\mathrm{H}]^{+},{ }^{37} \mathrm{Cl}\right)$.

(Z)-6-bromo-2-((2-phenylthiazol-4-yl)methylene)benzofuran-3(2H)-one $(\mathbf{2 g})$ : yellow powder, purified by column chromatography (eluent: dichlorometane: acetone $=25: 1$ ), Yield $=78 \%$; mp: $220.5-221.0^{\circ} \mathrm{C}$; $R_{\mathrm{f}}=0.5$ (eluent: dichloromethane: acetone $=25: 1 \mathrm{v} / \mathrm{v}$ ). IR: v( $\left.\mathrm{cm}^{-1}\right)$ : 2944.77 (C-H aromatic), $1602.56(\mathrm{C}=\mathrm{O})$.
${ }^{1} \mathrm{H}$ NMR $\left(600 \mathrm{MHz}, \mathrm{CDCl}_{3}\right) \delta 8.11(\mathrm{~s}, 1 \mathrm{H}, \mathrm{CH}-5$ thiazole), 8.04 - 8.03 (m, 2H, CH-2', CH-6'), 7.67 (d, $1 \mathrm{H}, \mathrm{CH}-4, J=8.1 \mathrm{~Hz}$ ), 7.55 (s, 1H, CH-7), $7.51-7.50$ (m, 3H, CH-3', CH-4', CH-5'), 7.38 (d, 1H, CH-5, $J=8.1 \mathrm{~Hz}), 7.31(\mathrm{~s}, 1 \mathrm{H}$, thiazole-CH=C $<) .{ }^{13} \mathrm{C} \mathrm{NMR}$ $\left(151 \mathrm{MHz}, \mathrm{CDCl}_{3}\right) \delta 183.0(\mathrm{C}, \mathrm{C}=\mathrm{O}), 167.9(\mathrm{C}, \mathrm{C}-2$ thiazole), 166.0 (C, C-8), 149.0 (C, C-4 thiazole), 147.5 (C, C-2), 132.9 (C, C-1'), 131.4 (C, C-6), 130.6 (CH, C-4'), 129.1 (CH, C-2', C-6'), 127.4 (CH, C-5), 126.8 (CH, C-3', C-5'), 125.6 (CH, C-4), $124.2(\mathrm{CH}, \mathrm{C}-5$ thiazole), 120.9 (C, C-9), $116.6(\mathrm{CH}, \mathrm{C}-7), 107.2(\mathrm{CH}$, thiazole-CH=C<). ESI ${ }^{+}-\mathrm{MS}: m / z 384.0\left([\mathrm{M}+\mathrm{H}]^{+},{ }^{81} \mathrm{Br}\right)$, $382.1\left([\mathrm{M}+\mathrm{H}]^{+},{ }^{79} \mathrm{Br}\right.$ ) (calculated for $\mathrm{C}_{18} \mathrm{H}_{11} \mathrm{BrNO}_{2} \mathrm{~S}$ $\left.383.96[\mathrm{M}+\mathrm{H}]^{+},{ }^{81} \mathrm{Br}, 381.99[\mathrm{M}+\mathrm{H}]^{+},{ }^{79} \mathrm{Br}\right)$.

(Z)-6-bromo-2-((2-(4-methoxyphenyl)thiazol-4-yl)methylene)benzofuran-3(2H)-one $(\mathbf{2 h})$ : bright-yellow powder, purified by column chromatography (eluent: dichlorometane: acetone $=25: 1)$, Yield $=86 \%$; $\mathrm{mp}$ : $222.1-222.8^{\circ} \mathrm{C} ; R_{\mathrm{f}}=0.5$ (eluent: dichloromethane: acetone $=25: 1 v / v)$. IR: $v\left(\mathrm{~cm}^{-1}\right): 3115.44(\mathrm{C}-\mathrm{H}$ aromatic $)$, $1596.77(\mathrm{C}=\mathrm{O}) .{ }^{1} \mathrm{H}$ NMR $\left(600 \mathrm{MHz}, \mathrm{CDCl}_{3}\right) \delta 8.04$ (s, 1H, CH-5 thiazole), 7.93 (d, 2H, CH-2', CH-6', $J=8.4 \mathrm{~Hz}$ ), 7.67 (d, 1H, CH-4, $J=8 \mathrm{~Hz}), 7.55$ (s, 1H, CH-7), 7.38 (d, 1H, CH-5, J=8 Hz), 7.23 (s, 1H, thiazole-CH=C<), 6.98 (d, 2H, CH-3', CH-5', $J=8.4$ $\mathrm{Hz}), 3.87$ (s, 3H, OCH 3$).{ }^{13} \mathrm{C} \mathrm{NMR}\left(151 \mathrm{MHz}, \mathrm{CDCl}_{3}\right)$ $\delta 183.0(\mathrm{C}, \mathrm{C}=\mathrm{O}), 167.8(\mathrm{C}, \mathrm{C}-2$ thiazole $), 165.9(\mathrm{C}$, C-8), 161.6 (C, C-4'), 148.9 (C, C-4 thiazole), 147.3 (C, C-2), 131.3 (C, C-1'), 128.3 (CH, C-2',C-6'), 127.4 (CH, C-5), 125.9 (C, C-6), 125.6 (CH, C-4), $123.6(\mathrm{CH}, \mathrm{C}-5$ thiazole), 121 (C, C-9), $116.6(\mathrm{CH}$, C-7), 114.4 (CH, C-3', C-5'), 107.4 (CH, thiazole$\mathbf{C H}=\mathrm{C}<), 55.5 \quad\left(\mathrm{C}-\mathrm{OCH}_{3}\right) . \quad \mathrm{ESI}^{+}-\mathrm{MS}: \mathrm{m} / z \quad 414.1$ $\left([\mathrm{M}+\mathrm{H}]^{+},{ }^{81} \mathrm{Br}\right), 412.1\left([\mathrm{M}+\mathrm{H}]^{+},{ }^{79} \mathrm{Br}\right)$ (calculated for $\left.\mathrm{C}_{19} \mathrm{H}_{13} \mathrm{BrNO}_{3} \mathrm{~S} 413.97[\mathrm{M}+\mathrm{H}]^{+81} \mathrm{Br}, 411.99[\mathrm{M}+\mathrm{H}]^{+79} \mathrm{Br}\right)$. (Z)-6-bromo-2-((2-(4-chlorophenyl)thiazol-4-yl)methylene)benzofuran-3(2H)-one (2i): orange powder, purified by column chromatography (eluent: dichlorometane: acetone $=25: 1)$, Yield $=71 \%$; $\mathrm{mp}: 238.8$ $239.8^{\circ} \mathrm{C} ; R_{\mathrm{f}}=0.5$ (eluent: dichloromethane:acetone $=$ 25:1 v/v). IR: $v\left(\mathrm{~cm}^{-1}\right): 3094.23$ (C-H aromatic), 1599.66 $(\mathrm{C}=\mathrm{O}) .{ }^{1} \mathrm{H}$ NMR $\left(600 \mathrm{MHz}, \mathrm{CDCl}_{3}\right) \delta 8.11(\mathrm{~s}, 1 \mathrm{H}$, CH-5 thiazole), 7.93 (d, 2H, CH-2', CH-6', J=8.1 Hz), 7.67 (d, 1H, CH-4, J = 8 Hz), 7.55 (s, 1H, CH-7), 7.44 (d, 2H, CH-3', CH-5', J= 8.1 Hz), 7.39 (d, 1H, CH-5, $J=8 \mathrm{~Hz}), 7.22(\mathrm{~s}, 1 \mathrm{H}$, thiazole-CH=C $<) .{ }^{13} \mathrm{C}$ NMR $\left(151 \mathrm{MHz}, \mathrm{CDCl}_{3}\right) \delta 182.9(\mathrm{C}, \mathrm{C}=\mathrm{O}), 166.6(\mathrm{C}, \mathrm{C}-2$ thiazole), 166 (C, C-8), 149.2 (C, C-4 thiazole), 147.5 (C, C-2), 136.6 (C, C-4'), 131.5 (C, C-1'), 134.4 (C, C-6), 129.3 (CH, C-2', C-6'), 127.9 (CH, C-3', C-5'), 127.5 (CH, C-5), 125.6 (CH, C-4), $124.3(\mathrm{CH}, \mathrm{C}-5$ thiazole), 120.9 (C, C-9), $116.6(\mathrm{CH}, \mathrm{C}-7), 106.9(\mathrm{CH}$, thiazole-CH=C<). ESI ${ }^{+}$-MS: $m / z$ $418.0\left([\mathrm{M}+\mathrm{H}]^{+},{ }^{35} \mathrm{Cl}\right.$, $\left.{ }^{79} \mathrm{Br}\right), 420.0\left([\mathrm{M}+\mathrm{H}]+{ }^{+35} \mathrm{Cl},{ }^{81} \mathrm{Br}\right.$ and $\left.{ }^{37} \mathrm{Cl},{ }^{79} \mathrm{Br}\right), 422.0$ $\left([\mathrm{M}+\mathrm{H}]+{ }^{+37} \mathrm{Cl},{ }^{81} \mathrm{Br}\right)$, (calculated for $\mathrm{C}_{18} \mathrm{H}_{10} \mathrm{BrClNO}_{2} \mathrm{~S}$ $417.93[\mathrm{M}+\mathrm{H}]+{ }^{+35} \mathrm{Cl},{ }^{79} \mathrm{Br}, 419.93[\mathrm{M}+\mathrm{H}]+{ }^{+35} \mathrm{Cl},{ }^{81} \mathrm{Br}$ and ${ }^{37} \mathrm{Cl},{ }^{79} \mathrm{Br}, 421.93[\mathrm{M}+\mathrm{H}]+{ }^{+37} \mathrm{Cl},{ }^{81} \mathrm{Br}$,). 
FARMACIA, 2020, Vol. 68, 3

(Z)-4,6-dimethoxy-2-((2-phenylthiazol-4-yl)methylene)benzofuran-3(2H)-one (2j): pale-yellow powder, purified by column chromatography (eluent: gradient of dichlorometane and dichlorometane:acetone = $25: 1)$, Yield $=72 \% ; \mathrm{mp}: 210.3-211^{\circ} \mathrm{C} ; R_{\mathrm{f}}=0.5$ (eluent: dichloromethane:acetone $=25: 1 \mathrm{v} / \mathrm{v})$. IR: $\mathrm{v}\left(\mathrm{cm}^{-1}\right)$ : 3003.59 (C-H aromatic), $1593.88(\mathrm{C}=\mathrm{O}) .{ }^{1} \mathrm{H}$ NMR $\left(600 \mathrm{MHz}, \mathrm{CDCl}_{3}\right) \delta 8.01-7.99(\mathrm{~m}, 3 \mathrm{H}, \mathrm{CH}-5$ thiazole, CH-2', CH-6'), 7.49 - 7.47 (m, 3H, CH-3', CH-4', CH-5'), 7.10 (s, 1H, thiazole- $\mathrm{CH}=\mathrm{C}<$ ), 6.38 (s, 1H, CH-7), 6.13 (s, 1H, CH-5), 3.94 (s, 3H, OCH 3 -C-4), 3.91 (s, 3H, OCH $3-\mathrm{C}-6) .{ }^{13} \mathrm{C} \mathrm{NMR}\left(151 \mathrm{MHz}, \mathrm{CDCl}_{3}\right)$ $\delta 180.1(\mathrm{C}, \mathrm{C}=\mathrm{O}), 169.1(\mathrm{C}, \mathrm{C}-6), 168.9(\mathrm{C}, \mathrm{C}-4)$, 167.6 (C, C-2 thiazole), 159.5 (C, C-8), 149.6 (C, C-4 thiazole), 148.6 (C, C-2), 133.1 (C, C-1'), 130.4 (CH, C-4'), 129.0 (CH, C-3', C-5'), 126.7 (CH, C-2', C-6'), 122.5 (CH, C-5 thiazole), 105.4 (C, C-9), 104.3 $(\mathrm{CH}$, thiazole- $\mathrm{CH}=\mathrm{C}<), 94.1(\mathrm{CH}, \mathrm{C}-5), 89.4(\mathrm{CH}$, C-7), $56.2\left(\mathrm{OCH}_{3}-\mathrm{C}-4\right), 56.1\left(\mathrm{OCH}_{3}-\mathrm{C}-6\right) . \mathrm{ESI}^{+}-\mathrm{MS}$ : $m / z 366.1$ (calculated for $\mathrm{C}_{20} \mathrm{H}_{16} \mathrm{NO}_{4} \mathrm{~S} 366.07[\mathrm{M}+\mathrm{H}]^{+}$). (Z)-4,6-dimethoxy-2-((2-(4-methoxyphenyl)thiazol4-yl)methylene)benzofuran-3(2H)-one $(2 \mathrm{k})$ : yellow powder, purified by column chromatography (eluent: gradient of dichlorometane:acetone $=25: 1$ and dichlorometane:acetone $=9: 1)$, Yield $=74 \%$; $\mathrm{mp}$ : $214.6-215.3^{\circ} \mathrm{C} ; R_{\mathrm{f}}=0.5$ (eluent: dichloromethane: acetone $=25: 1 \mathrm{v} / \mathrm{v})$. IR: $\mathrm{v}\left(\mathrm{cm}^{-1}\right): 2989.12(\mathrm{C}-\mathrm{H}$ aromatic), $1593.88(\mathrm{C}=\mathrm{O}) .{ }^{1} \mathrm{H}$ NMR $\left(600 \mathrm{MHz}, \mathrm{CDCl}_{3}\right) \delta 7.94-$ 7.92 (m, 3H, CH-2 thiazole, CH-2', CH-6'), 7.07 (s, $1 \mathrm{H}$, thiazole- $\mathrm{CH}=\mathrm{C}<), 6.96(\mathrm{~d}, 2 \mathrm{H}, \mathrm{CH}-3$ ', $\mathrm{CH}-5$ ', $J=8.8 \mathrm{~Hz}$ ), 6.37 (s, 1H, CH-7), 6.12 (s, 1H, CH-5), 3.94 (s, 3H, OCH ${ }_{3}-\mathrm{C}-4$ ), 3.91 (s, 3H, OCH $\left.3-\mathrm{C}-6\right)$, 3.86 (s, 3H, OCH $3-\mathrm{C}-4$ '). ${ }^{13} \mathrm{C}$ NMR (151 MHz, $\left.\mathrm{CDCl}_{3}\right)$

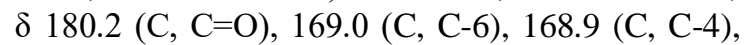
167.4 (C, C-2 thiazole), 161.4 (C, C-4'), 159.4 (C, C-8), 149.3 (C, C-4 thiazole), 148.5 (C, C-2), 128.2 (CH, C-2', C-6'), 126.1 (C, C-1'), 121.8 (CH, C-5 thiazole), 114.3 (CH, C-3', C-5'), 105.4 (C, C-9), 104.4 $(\mathrm{CH}$, thiazole- $\mathrm{CH}=\mathrm{C}<), 94.1(\mathrm{CH}, \mathrm{C}-5), 89.4(\mathrm{CH}$, $\mathrm{C}-7), 56.2\left(\mathrm{OCH}_{3}-\mathrm{C}-4\right), 56.1\left(\mathrm{OCH}_{3}-\mathrm{C}-6\right), 55,4$ $\left(\mathrm{OCH}_{3}-\mathrm{C}-4^{\prime}\right)$. ESI ${ }^{+}-\mathrm{MS}$ : $m / z 396.1$ (calculated for $\left.\mathrm{C}_{21} \mathrm{H}_{18} \mathrm{NO}_{5} \mathrm{~S} 396.08[\mathrm{M}+\mathrm{H}]^{+}\right)$.

(Z)-2-((2-(4-chlorophenyl)thiazol-4-yl)methylene)-4,6dimethoxybenzofuran-3(2H)-one (2l): pale-yellow powder, purified by column chromatography (eluent: gradient of dichlorometane and dichlorometane:acetone = 25:1), Yield $=80 \% ; \mathrm{mp}: 234.3-235.8^{\circ} \mathrm{C} ; R_{\mathrm{f}}=0.5$ (eluent: dichloromethane:acetone $=25: 1 \mathrm{v} / \mathrm{v})$. IR: $v\left(\mathrm{~cm}^{-1}\right)$ : 3015.16 (C-H aromatic), 1590.99 (C=O). ${ }^{1} \mathrm{H}$ NMR $\left(600 \mathrm{MHz}, \mathrm{CDCl}_{3}\right) \delta 8.00$ (s, 1H, CH-5 thiazole), 7.92 (d, 2H, CH-2', CH-6', J = 8.3 Hz), 7.43 (d, 2H, CH-3', $\mathrm{CH}^{-5}$ ', $\left.J=8.3 \mathrm{~Hz}\right), 7.08(\mathrm{~s}, 1 \mathrm{H}$, thiazole- $\mathrm{CH}=\mathrm{C}<$ ), 6.39 (s, 1H, CH-7), 6.15 (s, 1H, CH-5), 3.96 (s, 3H, $\left.\mathrm{OCH}_{3}-\mathrm{C}-4\right), 3.92$ (s, 3H, OCH $\left.3-\mathrm{C}-6\right) .{ }^{13} \mathrm{C}$ NMR (151 $\left.\mathrm{MHz}, \mathrm{CDCl}_{3}\right) \delta 180.1$ (C, C=O), 169.1 (C, C-6), 168.9 (C, C-4), 166.2 (C, C-2 thiazole), 159.6 (C, C-8), 149.8 (C, C-4 thiazole), 148.7 (C, C-2), 136.4 (C, C-4'), 131.6 (C, C-1'), 129.3 (CH, C-3', C-5'), 127.9 (CH,
C-2', C-6'), 122.6 (CH, C-5 thiazole), 105.4 (C, C-9), $104.0(\mathrm{CH}$, thiazole- $\mathrm{CH}=\mathrm{C}<), 94.2(\mathrm{CH}, \mathrm{C}-5), 89.5(\mathrm{CH}$, $\mathrm{C}-7), 56.3\left(\mathrm{OCH}_{3}-\mathrm{C}-4\right), 56.2\left(\mathrm{OCH}_{3}-\mathrm{C}-6\right) . \mathrm{ESI}^{+}-\mathrm{MS}$ : $m / z 400.00\left([\mathrm{M}+\mathrm{H}]^{+35} \mathrm{Cl}\right), 402.00\left([\mathrm{M}+\mathrm{H}]^{+37} \mathrm{Cl}\right)$ (calculated for $\mathrm{C}_{20} \mathrm{H}_{15} \mathrm{ClNO}_{4} \mathrm{~S} 400.03[\mathrm{M}+\mathrm{H}]{ }^{+},{ }^{35} \mathrm{Cl}, 402.03[\mathrm{M}+\mathrm{H}]^{+}$, $\left.{ }^{37} \mathrm{Cl}\right)$.

(Z)-6-methyl-2-((2-phenylthiazol-4-yl)methylene)benzofuran-3(2H)-one $(\mathbf{2 m})$ : bright-yellow powder, purified by column chromatography (eluent: dichlorometane: acetone $=25: 1)$, Yield $=82 \% ; \mathrm{mp}: 200.8$ $201.5^{\circ} \mathrm{C} ; R_{\mathrm{f}}=0.5$ (eluent: dichloromethane:acetone $=$ 25:1 v/v). IR: $v\left(\mathrm{~cm}^{-1}\right): 3128.94$ (C-H aromatic), 1593.88 $(\mathrm{C}=\mathrm{O}) .{ }^{1} \mathrm{H}$ NMR $\left(600 \mathrm{MHz}, \mathrm{CDCl}_{3}\right) \delta 8.10(\mathrm{~s}, 1 \mathrm{H}$, $\mathrm{CH}-5$ thiazole), 7.99 - 8.01 (m, 2H, CH-2', $\mathrm{CH}_{-6}$ '), 7.68 (d, 1H, CH-4, J = 7.8 Hz), $7.49-7.44$ (m, 3H, CH-3', CH-4', CH-5'), 7.20 (s, 1H, thiazole-CH=C<), 7.13 (s, 1H, CH-7), 7.03 (d, 1H, CH-5, J = $7.8 \mathrm{~Hz}$ ), 2.50 (s, 3H, CH 3$\left.).{ }^{13} \mathrm{C} \mathrm{NMR} \mathrm{(151} \mathrm{MHz,} \mathrm{CDCl}_{3}\right) \delta$ 183.8 (C, C=O), 167.7 (C, C-2 thiazole), 166.5 (C, C-8), 149.5 (C, C-4 thiazole), 149.2 (C, C-2), 148.0 (C, C-6), 133.0 (C, C-1'), 130.5 (CH, C-4'), 129.1 (CH, C-3', C-5'), 126.7 (CH, C-2', C-6'), 125.0 (CH, C-5), 124.4 (CH, C-4), 123.4 (CH, C-5 thiazole), 119.5 (C, C-9), $113.1(\mathrm{CH}, \mathrm{C}-7), 105.9(\mathrm{CH}$, thiazole- $\mathrm{CH}=\mathrm{C}<$ ), $22.7\left(\mathrm{CH}_{3}\right)$. ESI ${ }^{+}-\mathrm{MS}: m / 2320.1$ (calculated for $\mathrm{C}_{19} \mathrm{H}_{14} \mathrm{NO}_{2} \mathrm{~S}$ $\left.320.07[\mathrm{M}+\mathrm{H}]^{+}\right)$.

(Z)-2-((2-(4-methoxyphenyl)thiazol-4-yl)methylene)6-methylbenzofuran-3(2H)-one $(\mathbf{2 n})$ : yellow powder, purified by column chromatography (eluent: dichlorometane: acetone $=25: 1)$, Yield $=80 \% ; \mathrm{mp}: 204.0$ $204.9^{\circ} \mathrm{C} ; R_{\mathrm{f}}=0.5$ (eluent: dichloromethane:acetone $=$ 25:1 v/v). IR: $v\left(\mathrm{~cm}^{-1}\right): 2958.27$ (C-H aromatic), 1608.34 $(\mathrm{C}=\mathrm{O}) .{ }^{1} \mathrm{H}$ NMR $\left(600 \mathrm{MHz}, \mathrm{CDCl}_{3}\right) \delta 8.04(\mathrm{~s}, 1 \mathrm{H}$, CH-5 thiazole), 7.94 (d, 2H, CH-2', CH-6', $J=8.8$ $\mathrm{Hz}), 7.69$ (d, 1H, CH-4, J = $7.8 \mathrm{~Hz}), 7.18(\mathrm{~s}, 1 \mathrm{H}$, thiazole-CH=C<), $7.13(\mathrm{~s}, 1 \mathrm{H}, \mathrm{CH}-7), 7.04(\mathrm{~d}, 1 \mathrm{H}$, $\mathrm{CH}-5, J=7.8 \mathrm{~Hz}$ ), 6.98 (d, 2H, CH-3', CH-5', $J=8.8$ $\mathrm{Hz}), 3.88$ (s, 3H, $\left.\mathrm{OCH}_{3}\right), 2.51\left(\mathrm{~s}, 3 \mathrm{H}, \mathrm{CH}_{3}\right) .{ }^{13} \mathrm{C} \mathrm{NMR}$ $\left(151 \mathrm{MHz}, \mathrm{CDCl}_{3}\right) \delta 183.8(\mathrm{C}, \mathrm{C}=\mathrm{O}), 167.5(\mathrm{C}, \mathrm{C}-2$ thiazole), 166.5 (C, C-8), 161.4 (C, C-4'), 149.2 (C, C-4 thiazole), 149.0 (C, C-2), 147.9 (C, C-6), 128.2 (CH, C-2', C-6'), 125.9 (C, C-1'), 124.9 (CH, C-5), 124.3 (CH, C-4), 122.7 (CH, C-5 thiazole), 119.5 (C, C-9), 114.3 (CH, C-3', C-5'), 112.9 (CH, C-7), 105.9 $(\mathrm{CH}$, thiazole- $\mathrm{CH}=\mathrm{C}<), 55.4\left(\mathrm{OCH}_{3}-\mathrm{C}-4^{\prime}\right), 22.58\left(\mathrm{CH}_{3}-\right.$ C-6). ESI'-MS: $m / z 350.1$ (calculated for $\mathrm{C}_{19} \mathrm{H}_{14} \mathrm{NO}_{2} \mathrm{~S}$ $\left.350.08[\mathrm{M}+\mathrm{H}]^{+}\right)$.

(Z)-2-((2-(4-chlorophenyl)thiazol-4-yl)methylene)-6methylbenzofuran-3(2H)-one (2o): pale-orange powder, purified by column chromatography (eluent: dichlorometane: acetone $=50: 1)$, Yield $=84 \% ; \mathrm{mp}: 238.1$ $239.7^{\circ} \mathrm{C} ; R_{\mathrm{f}}=0.5$ (eluent: dichloromethane:acetone = 50:1 v/v). IR: $v\left(\mathrm{~cm}^{-1}\right): 2921.63$ (C-H aromatic), 1593.88 $(\mathrm{C}=\mathrm{O}) .{ }^{1} \mathrm{H}$ NMR $\left(600 \mathrm{MHz}, \mathrm{CDCl}_{3}\right) \delta 8.13(\mathrm{~s}, 1 \mathrm{H}$, CH-5 thiazole), 7.95 (d, 2H, CH-2', CH-6', J=8.4 Hz), 7.71 (d, 1H, CH-4, J = 7.8 Hz), 7.46 (d, 2H, CH-3', $\mathrm{CH}-5$ ', $J=8.4 \mathrm{~Hz}), 7.19(\mathrm{~s}, 1 \mathrm{H}$, thiazole-CH=C<), 7.15 (s, 1H, CH-7), 7.07 (d, 1H, CH-5, J= $7.8 \mathrm{~Hz}$ ), 
FARMACIA, 2020, Vol. 68, 3

$2.53\left(\mathrm{~s}, 3 \mathrm{H}, \mathrm{CH}_{3}\right) .{ }^{13} \mathrm{C} \mathrm{NMR}\left(151 \mathrm{MHz}, \mathrm{CDCl}_{3}\right) \delta 183.8$ (C, C=O), 166.6 (C, C-2 thiazole), 166.3 (C, C-8), 149.7 (C, C-4 thiazole), 149.3 (C, C-2), 148.2 (C, C-6), 136.5 (C, C-1'), 131.6 (C, C-4'), 129.3 (CH, C-3', C-5'), 127.9 (CH, C-2', C-6'), 125.1 (CH, C-5), 124.4 (CH, C-4), 123.5 (CH, C-5 thiazole), 119.5 (C, C-9), $113.1(\mathrm{CH}, \mathrm{C}-7), 105.6(\mathrm{CH}$, thiazole- $\mathrm{CH}=\mathrm{C}<), 22.7$ $\left(\mathrm{CH}_{3}-\mathrm{C}-6\right)$. ESI ${ }^{+}-\mathrm{MS}: \mathrm{m} / z 354.01\left([\mathrm{M}+\mathrm{H}]^{+},{ }^{35} \mathrm{Cl}\right), 356.01$ $\left([\mathrm{M}+\mathrm{H}]^{+},{ }^{37} \mathrm{Cl}\right)$ (calculated for $\mathrm{C}_{19} \mathrm{H}_{13} \mathrm{ClNO}_{2} \mathrm{~S} 354.03$ $\left.\left.[\mathrm{M}+\mathrm{H}]]^{+},{ }^{35} \mathrm{Cl}, 356.03[\mathrm{M}+\mathrm{H}]\right]^{+},{ }^{37} \mathrm{Cl}\right)$.

Biology

Cell cultures. Cancer cell models tested included both sensitive cells and their resistant counterparts. Their origins were previously reported. They included drugsensitive CCRF-CEM leukaemia and its multidrugresistant P-glycoprotein-over-expressing subline CEM/ ADR5000 [12, 17, 22], MDA-MB-231-pcDNA3 breast cancer cells and its resistant subline MDA-MB-231$B C R P$ clone 23 [11], HCT116 $\left(p 53^{+/+}\right)$, colon cancer cells and its knockout clone HCT116 ( $\left.p 53^{-/}\right)$, U87MG glioblastoma cells and its resistant subline U87MG.$\triangle E G F R$ [23-25]. To compare tumour with normal cells, HepG2 liver cancer cells and AML12 normal hepatocytes were used [23-25].

Cytotoxicity Assays. Resazurin reduction assay [35] was performed to assess the cytotoxicity of the thiazole aurones and doxorubicin as the control drug towards various sensitive and drug-resistant cancer cell lines, including the CCRF-CEM and CEM/ADR5000 leukaemia, MDA-MB231 breast cancer cells and its resistant subline MDA-MB231/BCRP, HCT116p $53^{+/+}$ colon cancer cells and its resistant subline HCT116 $p 53^{--}$, U87MG glioblastoma cells and its resistant subline U87MG. $\triangle E G F R$ and HepG2 hepatocarcinoma cells and normal AML12 hepatocytes. The assay is based on the reduction of the indicator dye, resazurin, to the highly fluorescent resorufin by viable cells. Non-viable cells rapidly lose their metabolic capacity to reduce resazurin and, thus, no longer produce fluorescent signals. Briefly, adherent cells were detached by treatment with $0.25 \%$ trypsin/EDTA (Invitrogen, Darmstadt Germany) and an aliquot of $1 \times 10^{4}$ cells was placed in each well of a 96-well cell culture plate (Thermo Scientific, Langenselbold, Germany) in a total volume of $200 \mu \mathrm{L}$. Cells were allowed to attach overnight and were then treated with different concentrations of compounds. For suspension cells, aliquots of $2 \times 10^{4}$ cells per well were seeded in 96-well-plates in a total volume of $100 \mu \mathrm{L}$. The studied compound was immediately added in varying concentrations in an additional $100 \mu \mathrm{L}$ of culture medium to obtain a total volume of $200 \mu \mathrm{L} /$ well. After $72 \mathrm{~h}$, resazurin (SigmaAldrich, Schnelldorf, Germany) $(20 \mu \mathrm{L}, 0.01 \% \mathrm{w} / \mathrm{v})$ in distilled water was added to each well and the plates were incubated at $37^{\circ} \mathrm{C}$ for $4 \mathrm{~h}$. Fluorescence was measured on an Infinite M2000 ProTM plate reader (Tecan, Crailsheim, Germany) using an excitation wavelength of $544 \mathrm{~nm}$ and an emission wavelength of
$590 \mathrm{~nm}$. Each assay was done at least twice with six replicates each. The viability was evaluated based on a comparison with untreated cells. $\mathrm{IC}_{50}$ values represent the compound concentrations required to inhibit 50\% of cell proliferation and were calculated from a calibration curve. The presented $\mathrm{IC}_{50}$ are expressed as an average value of determinations, where the extreme values are found in $\mathrm{a} \pm 10 \%$ interval of the resulted average value.

Molecular Docking. The topoisomerases used as targets in the molecular docking study were taken from Protein Data Bank (PDB - www.rcsb.org). Both proteins were isolated from Homo sapiens and their three dimensional structures were obtained after X-ray diffraction. For topoisomerase I (PDB entry code 1SC7) the Cartesian coordinates of the centre of the search space were set to $\mathrm{x}=98.714, \mathrm{y}=1.491, \mathrm{z}=10.193$, while for topoisomerase II (PDB entry code $4 \mathrm{GOU}$ ) coordinates of the centre of the search space were set to $\mathrm{x}=$ $31.335, y=90.761, z=48.009[40,43]$. The binding sites were identified, thanks to the co-crystallized ligands in the original macromolecule structures from PDB, using AutoDock Tools 1.5.6 [34]. The search space was considered as cube, with the length of the sides $x=y=z=60$, for both targets, to ensure similar experimental conditions.

The processing of the files containing the ligands and the targets was performed according to the previous reported protocol $[32,41]$. The molecular docking study was carried using AutoDock 4.2 [34] in batch mode using in-house written scripts. 200 docked poses were generated for each ligand. Visualization and analysis of the docking results were performed using UCSF Chimera [36]. The sequence homology analysis of the two proteins was performed using Clustal Omega [38].

\section{Results and Discussion}

\section{Chemistry}

Data from literature describes various methods for the synthesis of aurones, using ortho-hydroxychalcones as starting material, by an oxidative cyclization reaction with different oxidizing agents: copper(II) acetate [3], hydrogen peroxide [37], selenium dioxide [37] and mercury(II) acetate [13].

The thiazole ortho-hydroxychalcones 1a-o were obtained as previously reported by our group, via a ClaisenSchmidt condensation of thiazole aldehydes with orthohydroxyacetophenones in an alkaline environment $[4,8,31,39,45]$.

In order to choose the optimal method for the synthesis of the desired products 2a-o, an initial screening of methods was performed to obtain compound 2a using as starting material compound 1a. An overview of the results that we obtained applying the methods stated above for the synthesis of compound 2a is presented in Figure 2, which shows that different reaction products were formed. Their structures were elucidated based 
on spectral analysis, as presented in the Materials and Methods section.

When copper(II) acetate was used as the oxidizing agent and dimethylsulfoxide (DMSO) as solvent, the formation of both the aurone $\mathbf{2 a}$ and the corresponding flavone $\mathbf{3 a}$ in an approximate 1:1 molar ratio took place (Figure 2).

Next, when applying the oxidative cyclization of orthohydroxychalcones with hydrogen peroxide with alkaline catalysis, we noticed the formation of the corresponding hydroxyflavone 4a (Figure 2). In the literature, this method is reported for the synthesis of aurones or hydroxyflavones [37, 39].
Another oxidizing agent used to convert orthohydroxychalcones to flavonoid derivatives mentioned in literature is selenium dioxide [37]. In our case, the cyclization of the thiazole ortho-hydroxychalcones with selenium dioxide leads to hydroxyflavone $\mathbf{4 a}$ and flavone 3a in an approximate 1:1 molar ratio (Figure 2). The results of this screening showed that the best method for the synthesis of thiazole aurones is the oxidative cyclization of thiazole ortho-hydroxychalcones with mercury(II) acetate in pyridine (Figure 2). We applied this method for the synthesis of the whole new series of thiazole aurones 2a-o (Figure 3, Table I).

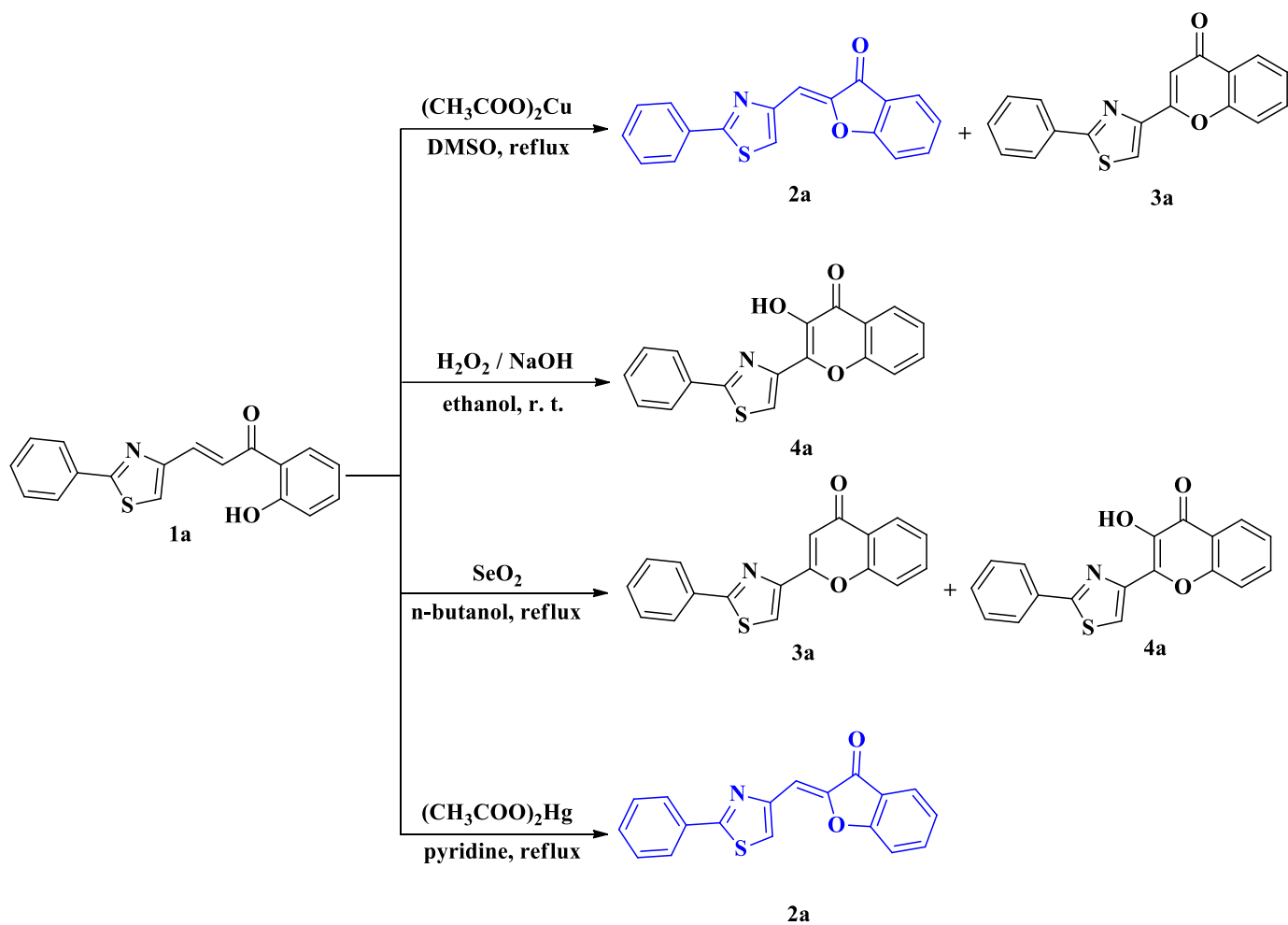

Figure 2.

The screening of the oxidative cyclization of 2-phenylthiazole ortho-hydroxychalcone 1a with different oxidizing agents in order to obtain the desired thiazole aurone $\mathbf{2 a}$

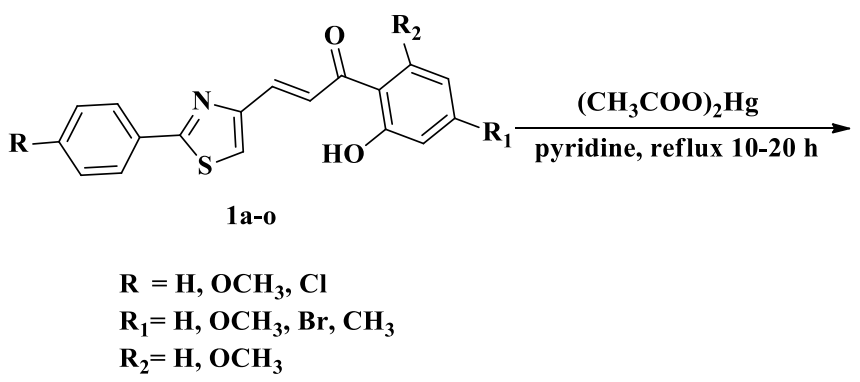<smiles>[R]c1ccc(-c2nc(/C=C3\Oc4cc([R7])cc([R2])c4C3=O)cs2)cc1</smiles>

2a-o

Figure 3.

The oxidative cyclization of thiazole ortho-hydroxychalcones with mercury(II) acetate in pyridine 


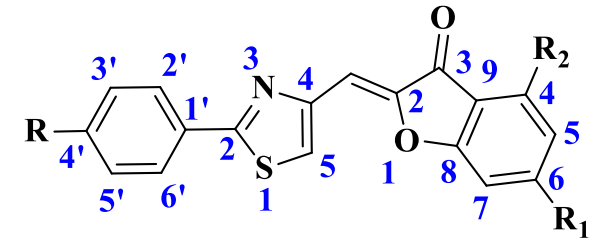

\begin{tabular}{ccccc}
\hline Compound & $\mathbf{R}$ & $\mathbf{R}_{\mathbf{1}}$ & $\mathbf{R}_{\mathbf{2}}$ & Yield (\%) \\
\hline $\mathbf{2 a}$ & $\mathrm{H}$ & $\mathrm{H}$ & $\mathrm{H}$ & 80 \\
$\mathbf{2 b}$ & $\mathrm{OCH}_{3}$ & $\mathrm{H}$ & $\mathrm{H}$ & 76 \\
$\mathbf{2}$ & $\mathrm{Cl}$ & $\mathrm{H}$ & $\mathrm{H}$ & 78 \\
$\mathbf{2}$ & $\mathrm{H}$ & $\mathrm{OCH}_{3}$ & $\mathrm{H}$ & 70 \\
$\mathbf{2}$ & $\mathrm{OCH}_{3}$ & $\mathrm{OCH}_{3}$ & $\mathrm{H}$ & 70 \\
$\mathbf{2}$ & $\mathrm{Cl}$ & $\mathrm{OCH}_{3}$ & $\mathrm{H}$ & 78 \\
$\mathbf{2}$ & $\mathrm{H}$ & $\mathrm{Br}$ & $\mathrm{H}$ & 86 \\
$\mathbf{2}$ & $\mathrm{Br}$ & $\mathrm{H}$ & 71 \\
$\mathbf{2}$ & $\mathrm{OCH}$ & $\mathrm{Br}$ & $\mathrm{H}$ & 72 \\
$\mathbf{2}$ & $\mathrm{Cl}$ & $\mathrm{OCH}_{3}$ & $\mathrm{OCH}_{3}$ & 80 \\
$\mathbf{2}$ & $\mathrm{H}$ & $\mathrm{OCH}_{3}$ & $\mathrm{OCH}_{3}$ & 82 \\
$\mathbf{2}$ & $\mathrm{OCH}_{3}$ & $\mathrm{CH}_{3}$ & $\mathrm{OCH}$ & 80 \\
$\mathbf{2}$ & $\mathrm{Cl}$ & $\mathrm{CH}_{3}$ & $\mathrm{H}$ & 84 \\
$\mathbf{2 n}$ & $\mathrm{H}$ & $\mathrm{CH}_{3}$ & $\mathrm{H}$ & $\mathrm{H}$ \\
$\mathbf{2 0}$ & $\mathrm{OCH}$ & $\mathrm{Cl}$ & \\
\hline
\end{tabular}

Previous studies reported that the oxidative cyclization of ortho-hydroxychalcones with mercury(II) acetate in pyridine leads exclusively to the formation of the thermodynamically more stable $Z$-aurones [13]. In our case, the NMR spectral data also indicates that the aurones, synthesized by the same procedure, are in the Z-isomeric form, as detailed below. According to the literature data, the chemical shifts of the exocyclic vinylic carbons are found at $104.0-112.8 \mathrm{ppm}$ in the case of Z-aurones and respectively 119.8 - $122.2 \mathrm{ppm}$ for $E$-aurones [13]. In our case, in the ${ }^{13} \mathrm{C} N M R$ spectra of the synthesized aurones, all exocyclic vinylic carbons have chemical shifts below $107.4 \mathrm{ppm}$, which indicates the $Z$ configuration. Moreover, in the ${ }^{1} \mathrm{H}$ NMR spectra of aurones, the vinylic protons appear at chemical shifts below $8 \mathrm{ppm}$, which is characteristic also for the $Z$ isomeric forms of aurones [13].

In the ${ }^{1} \mathrm{H}$ NMR spectra of the synthesized thiazole aurones there are characteristic signals for the aromatic, aliphatic and vinylic protons. The proton from the $5^{\text {th }}$ position of the thiazole ring appears as a singlet at 7.99 - $8.77 \mathrm{ppm}$. One characteristic signal corresponding to the vinylic proton appears as a singlet in the ${ }^{1} \mathrm{H}$ NMR spectra between 6.95 and 7.26 ppm.

The signals corresponding to the protons located on the benzene ring and thiazole ring are present in the aromatic region. In the case of the aurones substituted with methoxy or methyl groups, the corresponding signals of the aliphatic protons are present in the aliphatic area.

In the ${ }^{13} \mathrm{C}$ NMR spectra of the thiazole aurones, a characteristic signal at $180-207 \mathrm{ppm}$ indicates the presence of the carbonyl group from the benzofuranone moiety. In the case of thiazole aurones substituted with methoxy or methyl groups, the signals for the aliphatic carbons are present in the aliphatic area of the spectra. All aromatic signals, from both the benzene ring and the thiazole ring, are present in the spectra. The ESI-MS spectra reveal the presence of molecular ions in the positive ionization mode, $[\mathrm{M}+\mathrm{H}]^{+}$, for all the synthesized compounds.

In the FT-IR spectra of the synthesized aurones, the stretching vibration of the carbonyl group appears at $1609-1590 \mathrm{~cm}^{-1}$. The bands present in the region $3130-2912 \mathrm{~cm}^{-1}$ correspond to the stretching vibration of the aromatic and aliphatic $\mathrm{C}$-H bonds.

In the ${ }^{1} \mathrm{H}$ NMR spectra of the unexpected hydroxylflavone $4 \mathbf{a}$ the characteristic signals for the vinylic protons that were present in the spectra of the orthohydroxychalcone are no longer present, because the cyclization reaction took place. There is one characteristic signal for the hydroxyl group proton, signal that confirms the formation of the hydroxyflavone $\mathbf{4 a}$, as opposed to that of the corresponding aurone $\mathbf{2 a}$. In addition, in the ${ }^{13} \mathrm{C}$ NMR spectra of the hydroxyflavone $\mathbf{4 a}$, with the exception of one signal characteristic for the carbonyl group, all signals are present in the aromatic area, including the ones corresponding to C-2 and C-3 which are not aromatic.

In the ${ }^{1} \mathrm{H}$ NMR spectra of the unexpected compound 3a there is no characteristic signal for a phenolic hydroxylic proton, thus indicating that this compound is another cyclization product of the ortho-hydroxychalcone 1a, formed in the reaction medium along with the corresponding aurone 2a (Figure 2). The structure of compound 3a was elucidated based on our previous researches regarding the cyclization reaction of thiazole ortho-hydroxychalcones into their 
FARMACIA, 2020, Vol. 68, 3

corresponding flavonoid derivatives, in different reaction conditions [9]. Both ${ }^{1} \mathrm{H} \mathrm{NMR}$ and ${ }^{13} \mathrm{C} \mathrm{NMR}$ spectral data of compound $\mathbf{3} \mathbf{a}$ are entirely found in the spectral characterization of a thiazole flavone previously synthesized by our group in different reaction conditions [9] and this fact determined us to conclude that the reaction product is the flavone $\mathbf{3 a}$.

Biological activity

The cytotoxicity of novel thiazole aurones $\mathbf{2 a - 0}$ was evaluated in a panel of nine cancer cell lines including sensitive and drug resistant phenotypes, as well as in normal AML12 hepatocytes. Doxorubicin was used as reference drug, based on the similarity of mechanisms of action with the literature reported flavonoids $[6,20]$. Cell lines CEM/ADR5000, MDA-MB-231-BCRP, HCT116 (p53\%) and U87MG. $\triangle E G F R$ were used as the corresponding resistant counterpart for CCRFCEM, MDA-MB-231- $p c D N A$, HCT116 $\left(p 53^{+/+}\right)$, U87MG respectively. The determined $\mathrm{IC}_{50}$ values in the cytotoxic assay and the degree of resistance or the selectivity index are presented in Tables II-IV. The degree of resistance (DR) was determined as the ratio of $\mathrm{IC}_{50}$ value in the resistant type divided by the $\mathrm{IC}_{50}$ in the sensitive cell line. The selectivity index (SI) was determined as the ratio of $\mathrm{IC}_{50}$ value in the normal AML12 hepatocytes divided by the $\mathrm{IC}_{50}$ in HepG2 hepatocarcinoma cells [33]. Irrelevant data, such as $\mathrm{IC}_{50}$ higher than $50 \mu \mathrm{M}$ were replaced with a hyphen. The related indexes which derived from these values were displayed as hyphen as well. The significant cytotoxic effect expressed as low $\mathrm{IC}_{50}$ values were highlighted in bold.

Overall, the highest cytotoxic effect was found against the leukaemia cells. In terms of activity, the results are moderate compared to doxorubicin, on the sensitive phenotype. Against the leukaemia cells doxorubicinresistant phenotype $\left(\mathrm{IC}_{50}=66.83 \pm 2.20 \mu \mathrm{M}\right)$, compound 2a showed the most promising result $\left(\mathrm{IC}_{50}=5.85 \pm\right.$
$0.46 \mu \mathrm{M})$, whereas against the sensitive phenotype, compound 2e showed the best result $\left(\mathrm{IC}_{50}=13.18 \pm\right.$ $1.03 \mu \mathrm{M})$, but still moderate, referred to the positive control $\left(\mathrm{IC}_{50}=0.02 \pm 0.00 \mu \mathrm{M}\right)$. For compound $\mathbf{2 a}$, the degree of resistance is low on leukaemia cells $(\mathrm{DR}=0.36)$, this value being the best for the compounds from the current series.

In terms of substitutions on this aurone's scaffold, compound 2a, the compound with the best cytotoxic activity on the leukaemia resistant cell lines, does not have any substituent on the phenylthiazole system, nor on the benzofuranone ring. The next best cytotoxic activities were also displayed by compounds that don't have any substituents on the benzofuranone ring, namely, compounds $\mathbf{2 b}$ and $\mathbf{2 c}$, compound $\mathbf{2 c}$ exhibiting better activity than compound $\mathbf{2 b}$. Compound $\mathbf{2 c}$ is substituted on the phenylthiazole system with the chlorine group, an electron withdrawing substituent, whereas compound $\mathbf{2 b}$ is substituted on the phenylthiazole system with the methoxy group, an electron donating substituent. These results could indicate that substitution with an electron donating group decreases the cytotoxic activity of the compounds and the substitution with an electron withdrawing group is favourable.

On the breast adenocarcinoma cells, the activity of compounds 2a-o is negligible, with the exception of compound $\mathbf{2} \mathbf{i}$, which exhibited the best activity against the MDA-MB231/BCRP strain $\left(\mathrm{IC}_{50}=5.43 \pm 3.17 \mu \mathrm{M}\right)$. Moreover, the activity of this compound against the MDA-MB231/BCRP resistant strain, in comparison to the MDA-MB231 sensitive strain is improved, having the degree of resistance of 0.11 .

In the structure of this compound, we can also find chlorine on the phenylthiazole system, as well as bromine, another electron withdrawing group, on the benzofuranone ring.

Cytotoxicity of thiazole aurones $\mathbf{2 a - 0}$ and doxorubicin towards the leukaemia cells and the breast adenocarcinoma cells, expressed as $\mathrm{IC}_{50}$ values $(\mu \mathrm{M})$, with the degree of resistance (DR)

\begin{tabular}{ccccccc}
\hline Samples & \multicolumn{3}{c}{ Leukaemia cells } & \multicolumn{3}{c}{ Breast adenocarcinoma cells } \\
\cline { 2 - 7 } 2a & CCRF-CEM & CEM/ADR5000 & DR & MDA-MB231 & MDA-MB231/BCRP & DR \\
$\mathbf{2 b}$ & $26.36 \pm 0.98$ & $\mathbf{5 . 8 5} \pm \mathbf{0 . 4 6}$ & $\mathbf{0 . 3 6}$ & - & - & - \\
$\mathbf{2} \mathbf{c}$ & $17.74 \pm 2.01$ & $13.48 \pm 0.88$ & 0.76 & - & - & - \\
$\mathbf{2 d}$ & $25.89 \pm 2.19$ & - & - & - & - \\
$\mathbf{2} \mathbf{e}$ & $13.18 \pm 1.03$ & $53.48 \pm 3.42$ & 1.42 & - & - & - \\
$\mathbf{2 f}$ & $29.62 \pm 3.11$ & $18.65 \pm 1.37$ & 0.88 & $36.04 \pm 2.15$ & - & - \\
$\mathbf{2 g}$ & $28.15 \pm 1.56$ & $26.03 \pm 2.11$ & 1.23 & - & - & - \\
$\mathbf{2 h}$ & $16.21 \pm 1.24$ & $34.60 \pm 3.29$ & 2.61 & - & - & - \\
$\mathbf{2 i}$ & $21.05 \pm 1.95$ & $42.33 \pm 3.42$ & 1.65 & $51.11 \pm 3.09$ & $\mathbf{5 . 4 3} \pm \mathbf{3 . 1 7}$ & $\mathbf{0 . 1 1}$ \\
$\mathbf{2 j}$ & - & - & - & - & - \\
$\mathbf{2 k}$ & $28.61 \pm 1.94$ & $44.29 \pm 2.84$ & 1.55 & - & - & - \\
$\mathbf{2}$ & - & - & - & $22.27 \pm 1.07$ & - & - \\
$\mathbf{2} \mathbf{2}$ & $22.14 \pm 1.09$ & $25.95 \pm 1.18$ & 1.17 & - & - & - \\
$\mathbf{2 n}$ & $39.68 \pm 2.67$ & $41.91 \pm 3.65$ & 1.06 & - & - & - \\
$\mathbf{2 0}$ & $18.13 \pm 1.89$ & $33.59 \pm 2.36$ & 1.85 & - & $\mathbf{0 . 0 7}$ & $\mathbf{0 . 0 0}$ \\
Doxorubicin & $\mathbf{0 . 0 2} \pm \mathbf{0 . 0 0}$ & $66.83 \pm 2.20$ & 3341 & $\mathbf{0 . 0 7 3} \pm \mathbf{0 . 1 0}$ & $\mathbf{6 . 1 4}$ \\
\hline
\end{tabular}


The anticancer activity of the novel synthesized compounds is overall more intense on the resistant

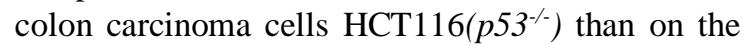
sensitive phenotype HCT116 $\left(p 53^{+/+}\right)$. Compound 2e and $\mathbf{2 n}$ showed the best results on the HCT116 $\left(p 53^{-/-}\right)$resistant phenotype $\left(\mathrm{IC}_{50}=15.67 \pm\right.$ $1.11 \mu \mathrm{M}$ and $14.55 \pm 1.31 \mu \mathrm{M}$, respectively). However, the $\mathrm{IC}_{50}$ values are higher than the one displayed by doxorubicin $\left(\mathrm{IC}_{50}=0.97 \pm 0.02 \mu \mathrm{M}\right)$.

These two compounds have a methoxy group on the phenylthiazole system, but they also have electron donating groups on the benzofuranone ring. Compound $\mathbf{2 e}$ is substituted with a methoxy group on the benzo-

furanone ring, whereas compound $\mathbf{2 n}$ is substituted with a methyl group on this moiety.

On the glioblastoma cells, compound $\mathbf{2 e}$ was the only one from our series which displayed a noticeable activity, yet the $\mathrm{IC}_{50}$ values are not comparable to the ones of the reference drug.

Compound $\mathbf{2 e}$ is also one of the only two compounds from our series that displayed cytotoxic activity against the hepatocarcinoma cells with a $\mathrm{IC}_{50}$ value of $18.75 \pm 1.17 \mu \mathrm{M}$.

Promisingly, all compounds manifest cytotoxic activity against the normal hepatocytes at high concentrations, reaching a selectivity index value of 5.84 for compound 2e.

Table III

Cytotoxicity of thiazole aurones 2a-o and doxorubicin towards the colon carcinoma cells and the glioblastoma cells, expressed as $\mathrm{IC}_{50}$ values $(\mu \mathrm{M})$, with the degree of resistance

\begin{tabular}{|c|c|c|c|c|c|c|}
\hline \multicolumn{7}{|c|}{ (DR) } \\
\hline \multirow[t]{2}{*}{ Samples } & \multicolumn{3}{|c|}{ Colon carcinoma cells } & \multicolumn{3}{|c|}{ Glioblastoma cells } \\
\hline & $\operatorname{HCT116}\left(p 53^{+/+}\right)$ & $\operatorname{HCT116}\left(p 53^{-/-}\right)$ & DR & U87MG & $\begin{array}{l}\text { U87MG. } \\
\text { AEGFR }\end{array}$ & DR \\
\hline $2 \mathbf{a}$ & - & - & - & - & - & - \\
\hline $2 b$ & - & - & - & - & - & - \\
\hline $2 c$ & - & $36.52 \pm 2.48$ & - & - & - & - \\
\hline 2d & - & $33.55 \pm 2.64$ & - & - & - & - \\
\hline $2 \mathrm{e}$ & - & $15.67 \pm 1.11$ & - & $27.38 \pm 1.67$ & $42.88 \pm 3.77$ & 1.57 \\
\hline $2 f$ & - & - & - & - & - & - \\
\hline $2 \mathrm{~g}$ & - & $26.35 \pm 1.97$ & - & - & - & - \\
\hline $2 \mathrm{~h}$ & - & $31.84 \pm 2.07$ & - & - & - & - \\
\hline $2 \mathrm{i}$ & - & $38.81 \pm 1.30$ & - & - & - & - \\
\hline $2 \mathrm{j}$ & - & - & - & - & - & - \\
\hline $2 \mathbf{k}$ & - & - & - & - & - & - \\
\hline 21 & - & $33.17 \pm 1.19$ & - & - & - & - \\
\hline $2 m$ & - & $29.08 \pm 1.45$ & - & - & - & - \\
\hline $2 n$ & - & $14.55 \pm 1.31$ & - & - & - & - \\
\hline 20 & - & $39.76 \pm 2.84$ & - & - & - & - \\
\hline Doxorubicin & $0.26 \pm 0.01$ & $0.97 \pm 0.02$ & 3.73 & $0.14 \pm 0.01$ & $0.53 \pm 0.08$ & 3.79 \\
\hline
\end{tabular}

Table IV

Cytotoxicity of thiazole aurones $\mathbf{2 a - 0}$ and doxorubicin towards the hepatocarcinoma cells and the normal hepatocytes, expressed as $\mathrm{IC}_{50}$ values $(\mu \mathrm{M})$, with the selectivity index (SI)

\begin{tabular}{cccc}
\hline Samples & \multicolumn{3}{c}{ Hepatic cells } \\
& HepG2 & AML12 & SI \\
\hline $\mathbf{2 a}$ & - & $>131.13$ & - \\
$\mathbf{2 b}$ & - & $>119.38$ & $>1.17$ \\
$\mathbf{2 c}$ & - & $>117.99$ & $>1.35$ \\
$\mathbf{2 d}$ & - & $>119.38$ & $>1.54$ \\
$\mathbf{2 e}$ & $18.75 \pm 1.17$ & $>109.56$ & $>5.84$ \\
$\mathbf{2 f}$ & $38.51 \pm 3.22$ & $>108.39$ & $>2.81$ \\
$\mathbf{2 g}$ & - & $>104.45$ & - \\
$\mathbf{2 h}$ & - & $>96.86$ & - \\
$\mathbf{2 i}$ & - & $>95.94$ & $>1.15$ \\
$\mathbf{2 j}$ & - & $>109.57$ & - \\
$\mathbf{2 k}$ & - & $>101.25$ & $>1.29$ \\
$\mathbf{2 l}$ & - & $>100.24$ & - \\
$\mathbf{2 m}$ & - & $>125.36$ & $>1.61$ \\
$\mathbf{2 n}$ & - & $>114.59$ & $>1.08$ \\
$\mathbf{2 0}$ & - & $>113.30$ & $>1.09$ \\
Doxorubicin & $\mathbf{2 . 1 5} \pm \mathbf{0 . 0 3}$ & $\mathbf{0 . 4 8}$ & $\mathbf{0 . 2 2}$ \\
\hline
\end{tabular}

\section{Molecular Docking}

The results of the molecular docking study evaluating the interactions between compounds $\mathbf{2 a - 0}$ and human topoisomerases I and II are presented in Table $\mathrm{V}$ as binding affinity expressed as the variation of the Gibbs free energy $(\Delta G)$, the consequent inhibition constant (Ki) and the energetically analysis and sterically dispersion of the predicted poses from the $2 \AA$ cluster containing the top binding conformation.

It is important to notice the amino acids which are in the vicinity of the studied binding site, because they could also influence the binding of compounds $\mathbf{2 a - 0}$. In the proximity of the $\mathrm{R}_{1}$ substituent, there are no amino acids with which it could interact; therefore its presence on the aurone's moiety does not influence the affinity for the enzyme. It may influence other parameters, such as lipophilicity, penetration or susceptibility to metabolism, but not the affinity for the enzyme. 
Table V

Binding affinity of the compounds 2a-o to the catalytic site of topoisomerases I and II expressed as variation of free Gibbs energy $(\mathrm{kcal} / \mathrm{mol})$ and inhibition constant $(\mu \mathrm{M})$. For the best pose of each compound, the analysis of the $2 \AA$ cluster of which it belongs is presented.

\begin{tabular}{|c|c|c|c|c|c|c|c|c|}
\hline \multirow[t]{3}{*}{ Compound } & \multicolumn{4}{|c|}{ Topoisomerase I } & \multicolumn{4}{|c|}{ Topoisomerase II } \\
\hline & \multirow[t]{2}{*}{$\Delta \mathrm{G} \mathrm{(kcal/mol)}$} & \multirow[t]{2}{*}{$\mathbf{K i}(\mu \mathrm{M})$} & \multicolumn{2}{|l|}{$2 \AA$ cluster } & \multirow[t]{2}{*}{$\Delta G(\mathrm{kcal} / \mathrm{mol})$} & \multirow[t]{2}{*}{$\mathbf{K i}(\boldsymbol{\mu M})$} & \multicolumn{2}{|l|}{$2 \AA$ cluster } \\
\hline & & & $\begin{array}{c}\text { Mean } \\
\Delta G(\text { kcal } / \mathrm{mol})\end{array}$ & NoC & & & $\begin{array}{c}\text { Mean } \\
\Delta G(\text { (kcal } / \mathrm{mol})\end{array}$ & NoC \\
\hline $2 \mathbf{a}$ & -7.38 & 3.89 & -7.33 & 31 & -5.87 & 49.80 & -5.76 & 6 \\
\hline $2 b$ & -6.73 & 11.66 & -6.58 & 16 & -5.81 & 55.11 & -5.72 & 57 \\
\hline $2 c$ & -7.16 & 5.64 & -6.74 & 28 & -5.75 & 60.98 & -5.59 & 70 \\
\hline 2d & -7.29 & 4.53 & -6.85 & 3 & -5.41 & 108.25 & -5.30 & 44 \\
\hline $2 e$ & -6.51 & 16.91 & -6.22 & 5 & -5.65 & 72.19 & -5.65 & 2 \\
\hline $2 f$ & -7.03 & 7.03 & -6.73 & 38 & -5.66 & 70.98 & -5.53 & 114 \\
\hline $2 \mathrm{~g}$ & -6.84 & 9.69 & -6.71 & 26 & -6.00 & 39.99 & -5.97 & 2 \\
\hline $2 \mathrm{~h}$ & -6.90 & 8.75 & -6.31 & 12 & -5.97 & 42.07 & -5.83 & 43 \\
\hline $2 \mathrm{i}$ & -7.36 & 4.03 & -6.90 & 20 & -6.01 & 39.32 & -5.88 & 120 \\
\hline $2 \mathbf{j}$ & -7.15 & 5.74 & -7.04 & 4 & -5.47 & 97.82 & -5.30 & 24 \\
\hline $2 k$ & -6.42 & 19.68 & -6.02 & 12 & -5.57 & 82.63 & -5.47 & 14 \\
\hline 21 & -6.69 & 12.48 & -6.40 & 12 & -5.47 & 97.82 & -5.32 & 13 \\
\hline $2 m$ & -7.22 & 5.10 & -7.18 & 10 & -5.81 & 55.11 & -5.64 & 21 \\
\hline $2 n$ & -6.76 & 11.09 & -6.55 & 5 & -5.99 & 40.67 & -5.86 & 62 \\
\hline 20 & -7.21 & 5.19 & -7.06 & 23 & -5.91 & 46.55 & -5.79 & 105 \\
\hline
\end{tabular}

$\Delta \mathrm{G}=$ variation of the free Gibbs energy, $\mathrm{Ki}=$ inhibition constant, NoC $=$ number of conformations

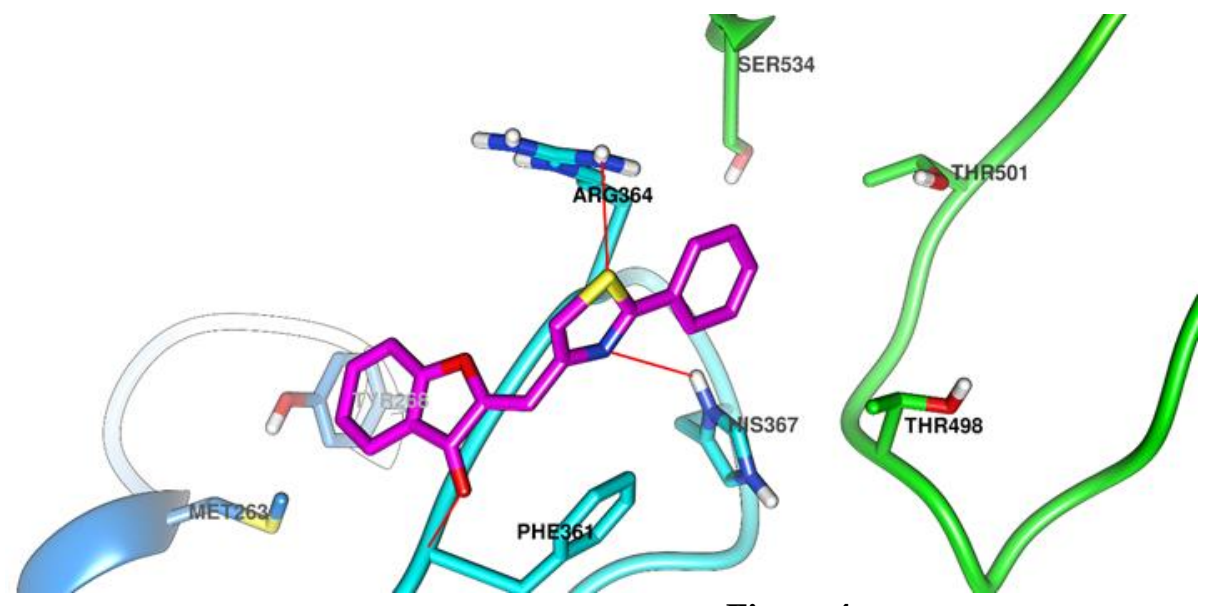

Figure 4

Compound $\mathbf{2 a}$ (carbon atoms depicted in magenta) bound to the human topoisomerase I

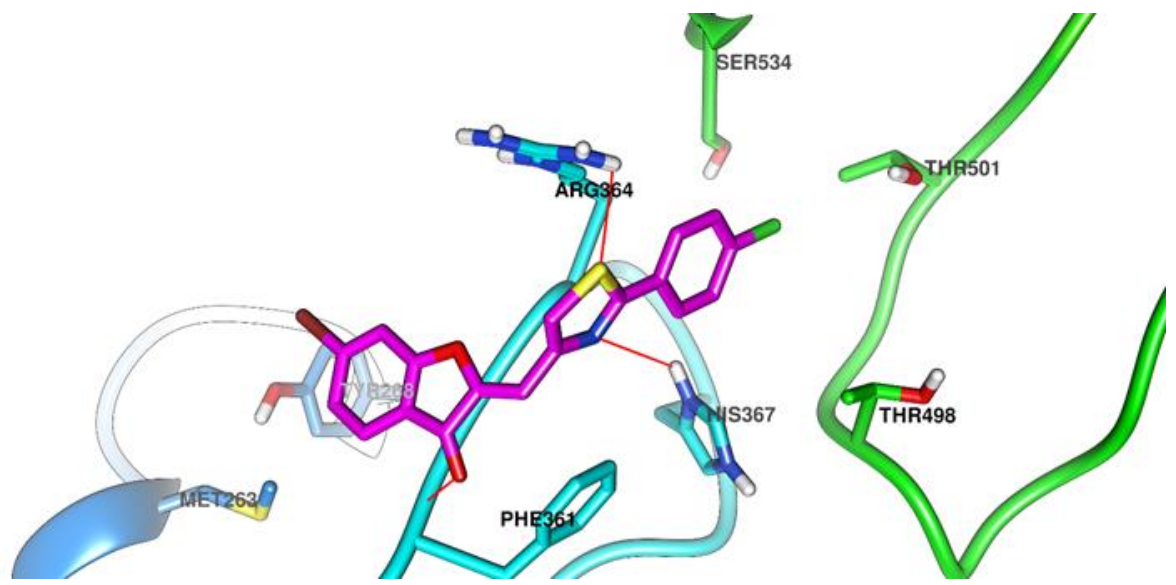

Figure 5.

Compound $\mathbf{2} \mathbf{i}$ (carbon atoms depicted in magenta) bound to the human topoisomerase I 
The presence of a substituent $\mathrm{R}_{2}$ on the aurone scaffold will determine the repulsion of the respective fragment from the Phe361-Arg362 peptide bridge, leading to a decrease in the binding affinity of a ligand substituted in this manner. This argument can explain why some compounds with a $\mathrm{R}_{2}$ substituent have reduced affinity for the enzyme (compounds $\mathbf{2} \mathbf{j}-\mathbf{l}$ ).

The phenyl fragment is oriented towards a pocket containing multiple polar amino acids (Thr498, Thr501, Ser534), but the distance between them is too big, so interaction with them is impossible, even if the nucleus carries a polar, but small substituent. The development of novel compounds targeting topoisomerase I could be directed to substitution of the phenyl nucleus with polar, but bulkier fragments in order to determine interaction with one of these amino acids with hydroxyl in their structure.

Regarding the interaction of our compounds with topoisomerase II, we can say that the binding affinity of compounds 2a-o is modest, indicating that this enzyme cannot be considered a target for the present series of compounds. Overall, binding affinity of the compounds is ranging between $\Delta \mathrm{G}=-6.01 \mathrm{kcal} / \mathrm{mol}$ for compound $2 \mathbf{i}$ and $\Delta \mathrm{G}=-5.41 \mathrm{kcal} / \mathrm{mol}$ for compound 2d.

The sequence homology analysis of the two topoisomerases revealed just a few conserved residues between the two structures. The $20.09 \%$ relative identity is concordant with the literature reports that suggest that topoisomerases have developed from different ancestral enzymes, followed different evolutionary paths and had some lateral gene transfer. This hypothesis could justify the large difference of binding affinity of our compounds for the two topoisomerases $[15,18]$.

\section{Conclusions}

A screening of methods regarding the optimal way to synthesize the new thiazole aurones 2a-o via the oxidative cyclization of ortho-hydroxychalcones was applied. The most convenient protocol is based on using mercury(II) acetate, because by applying this method, we got the highest amounts of desired products and the secondary by-products were found only in traces. The yields for obtaining the wanted thiazole aurones 2a-0 varied between $70 \%$ and $86 \%$. The structures of all synthesized compounds were confirmed by spectral analysis $1 \mathrm{D}$ NMR $\left({ }^{1} \mathrm{H},{ }^{13} \mathrm{C}\right), 2 \mathrm{D}$ NMR (COSY, HMQC), IR and MS.

Some of the newly synthesized thiazole aurones showed cytotoxic activity. Compound $\mathbf{2 a}$, the unsubstituted aurone, displayed good anticancer activity on the leukaemia resistant phenotype (CEM/ADR5000) and compound $\mathbf{2} \mathbf{i}$, substituted with chlorine on the phenylthiazole system and with bromine on the benzofuranone, displayed good anticancer activity on the breast adenocarcinoma cells, the resistant phenotype (MDA-MDB 231/BRCP). Compound 2e, which contains two metoxy groups, one on the phenyltiazole system and the other one on the benzofuranone, displayed the broadest cytotoxic spectra, with moderate activities, but presenting a good selectivity index.

Using the molecular docking study, the interactions between our compounds and topoisomerase I and II were evaluated. The evaluation of the results revealed the importance of the thiazole ring for establishing a hydrogen bond with His367 and Arg364. Moreover, the analysis of the docked poses revealed that a substitution on position 6 of the benzofuranone ring is unfavourable in terms of interaction with topoisomerase I. Regarding topoisomerase II, the molecular docking studies revelled that this is not the target of our compounds.

\section{Acknowledgement}

This research was funded by the "Iuliu Hațieganu" University of Medicine and Pharmacy Cluj-Napoca, Romania, by internal grant no. 5200/25 from 01.03.2018. VK is very grateful to the "Alexander von Humboldt" Foundation for the funding through the Linkage program (2015 - 2018); ATM is thankful to "Alexander von Humboldt" Foundation for an 18 months fellowship in Prof. Dr. Thomas Efferth's laboratory in Mainz, Germany through the "Georg Foster" Research Fellowship for Experienced Researcher program. The authors extend their appreciation and thanks to Prof. Dr. Thomas Efferth, Department of Pharmaceutical Biology, Institute of Pharmacy and Biochemistry, Johannes Gutenberg University, Mainz, Germany, for helping with the evaluation of the anticancer activity.

\section{Conflict of interest}

The authors declare no conflict of interest.

\section{References}

1. Aliabadi A, Shamsa F, Ostad SN, Emami S, Shafiee A, Davoodi J, Foroumadi A, Synthesis and biological evaluation of 2-phenylthiazole-4-carboxamide derivatives as anticancer agents. Eur J Med Chem., 2010; 45(11): 5384-5389.

2. Alsayari A, Muhsinah AB, Hassan MZ, Ahsan MJ, Alshehri JA, Begum N, Aurone: A biologically attractive scaffold as anticancer agent. Eur J Med Chem., 2019; 166: 417-431.

3. Ashok D, Ravi S, Ganesh A, Lakshmi BV, Adam S, Murthy SDS, Microwave-assisted synthesis and biological evaluation of carbazole-based chalcones, aurones and flavones. Med Chem Res., 2016; 25(5): 909-922.

4. Awoussong PK, Zaharia V, Ngameni B, Kuete V, Ntede HN, Fokunang CN, Abegaz BM, Ngadjui BT, Heterocycles 26: Synthesis, characterisation and anticancer activity of some thiazolic chalcones. Med Chem Res., 2015; 24(1): 131-141. 
5. Boumendjel A, Aurones: A Subclass of Flavones with Promising Biological Potential. Curr Med Chem., 2003; 10(23): 2621-2630.

6. Caroline FT, Oshiro C, Marsh S, Hernandez-Boussard T, McLeod H, Klein TE, Altman RB, Doxorubicin pathways: pharmacodynamics and adverse effects. Pharmacogenet Genom., 2011; 21(7): 440-446.

7. Cheng H, Zhang L, Liu Y, Chen S, Cheng H, Lu X, Zheng Z, Zhou GC, Design, synthesis and discovery of 5-hydroxyaurone derivatives as growth inhibitors against HUVEC and some cancer cell lines. Eur $J$ Med Chem., 2010; 45(12): 5950-5957.

8. Coman FM, Mbaveng A, Denisa L, Bencze LC, Vlase L, Imre S, Kuete V, Efferth T, Zaharia V, Heterocycles 44. Synthesis, characterization and anticancer activity of new thiazole ortho-hydroxychalcones. Med Chem Res., 2018; 27(Supplement C): 1396-1407.

9. Constantinescu T, Leonte D, Bencze LC, Vlase L, Imre S, Hanganu D, Zaharia V, Heterocycles 43. Synthesis, characterization and antioxidant activity of some thiazole hydroxychalcones and their flavonoidic derivatives. Farmacia, 2018; 66(4): 663-673.

10. Demirayak S, Yurttas L, Gundogdu-Karaburun N, Karaburun AC, Kayagil I, Synthesis and anti-cancer activity evaluation of newaurone derivatives. $J$ Enz Inhib Med Chem., 2015; 30(5): 816-825.

11. Doyle LA, Yang W, Abruzzo LV, Krogmann T, Gao Y, Rishi AK, Ross DD, A multidrug resistance transporter from human MCF-7 breast cancer cells. Proc Natl Acad Sci USA., 1998; 95(26): 1566515670.

12. Efferth T, Sauerbrey A, Olbrich A, Gebhart E, Rauch P, Weber HO, Hengstler JG, Halatsch ME, Volm M, Tew KD, Ross DD, Funk JO, Molecular modes of action of artesunate in tumour cell lines. $\mathrm{Mol}$ Pharmacol., 2003; 64(2): 382-394.

13. Elhadi AA, Osman H, Iqbal MA, Rajeswari S, Khadeer Ahamed MB, Abdul Majid AMS, Rosli MM, Razak IA, Majid ASA, Synthesis and structural elucidation of two new series of aurone derivatives as potent inhibitors against the proliferation of human cancer cells. Med Chem Res., 2015; 24(9): 3504-3515.

14. El-Messery SM, Hassan GS, Al-Omary FAM, ElSubbagh HI, Substituted thiazoles VI. Synthesis and antitumour activity of new 2-acetamido- and 2 or 3-propanamido-thiazole analogs. Eur J Med Chem., 2012; 54: 615-625.

15. Forterre P, Gadelle D, Phylogenomics of DNA topoisomerases: their origin and putative roles in the emergence of modern organisms. Nucleic Acids Res., 2009; 37(3): 679-692.

16. Ghorab M, Al-Said M, Antitumour activity of novel pyridine, thiophene and thiazole derivatives. Arch Pharm Res., 2012; 35(6): 965-973.

17. Gillet PJ, Efferth T, Steinbach D, Hamels J, de Longueville F, Bertholet V, Remacle J, Microarraybased detection of multidrug resistance in human tumour cells by expression profiling of ATP-binding cassette transporter genes. Cancer Res., 2004; 64(24): 8987-8993.

18. Goffart S, Hangas A, Pohjoismäki JLO, Twist and Turn-Topoisomerase Functions in Mitochondrial DNA Maintenance. Int J Mol Sci., 2019; 20(8): 2041: 1-17.
19. Hassan GS, El-Messery SM, Al-Omary FAM, ElSubbagh HI, Substituted thiazoles VII. Synthesis and antitumour activity of certain 2-(substituted amino)-4-phenyl-1,3-thiazole analogs. Bioorg Med Chem Lett., 2012; 22(20): 6318-6323.

20. Hassan GS, Georgey HH, George RF, Mohamed ER, Aurones and furoaurones: Biological activities and synthesis. Bull Fac Pharm Cairo Univ., 2018; 56(2): 121-127.

21. Huang W, Liu MZ, Li Y, Tan Y, Yang GF, Design, syntheses, and antitumour activity of novel chromone and aurone derivatives. Bioorg Med Chem., 2007; 15(15): 5191-5197.

22. Kimmig A, Gekeler V, Neumann M, Frese G, Kardos G, Diddens H, Niethammer D, Susceptibility of multidrug-resistant human leukemia cell lines to human interleukin 2-activated killer cells. Cancer Res., 1990; 50(21): 6793-6799.

23. Kuete V, Sandjo L, Nantchouang Ouete J, Fouotsa $\mathrm{H}$, Wiench B, Efferth T, Cytotoxicity and modes of action of three naturally occuring xanthones (8hydroxycudraxanthone $\mathrm{G}$, morusignin $\mathrm{I}$ and cudraxanthone I) against sensitive and multidrugresistant cancer cell lines. Phytomedicine, 2013; 21(3): 315-322.

24. Kuete V, Sandjo LP, Wiench B, Efferth T, Cytotoxicity and modes of action of four Cameroonian dietary spices ethno-medically used to treat Cancers: Echinops giganteus, Xylopia aethiopica, Imperata cylindrica and Piper capense. J Ethnopharmacol., 2013; 149(1): 245-253.

25. Kuete V, Tchakam PD, Wiench B, Ngameni B, Wabo HK, Tala MF, Moungang ML, Ngadjui BT, Murayama $\mathrm{T}$, Efferth T, Cytotoxicity and modes of action of four naturally occuring benzophenones: 2,2',5,6'tetrahydroxybenzophenone, guttiferone $\mathrm{E}$, isogarcinol and isoxanthochymol. Phytomedicine, 2013; 20(6): 528-536.

26. Lawrence NJ, Rennison D, McGown AT, Hadfield JA, The total synthesis of an aurone isolated from Uvaria hamiltonii: aurones and flavones as anticancer agents. Bioorg Med Chem Lett., 2003; 13(21): 3759-3763.

27. Liang X, Wu Q, Luan S, Yin Z, He C, Yin L, Zou Y, Yuan Z, Li L, Song X, He M, Lv C, Zhang W, A comprehensive review of topoisomerase inhibitors as anticancer agents in the past decade. Eur J Med Chem., 2019; 171: 129-168.

28. Lipinski CA, Lombardo F, Dominy BW, Feeney PJ, Experimental and computational approaches to estimate solubility and permeability in drug discovery and development settings. Adv Drug Deliver Rev., 2001; 46(1-3): 3-26

29. López-Lázaro M, Martin-Cordero C, Toro MV, Ayuso MJ, Flavonoids as DNA Topoisomerase I Poisons. J Enzyme Inhib Med Chem., 2002; 17(1): 25-29.

30. López-Lázaro M, Willmore E, Austin CA, The dietary flavonoids myricetin and fisetin act as dual inhibitors of DNA topoisomerases I and II in cells. Mutat Res., 2010; 696(1): 41-47.

31. Mager S, Zaharia V, Horn M, Simiti I, Heterocyclen 69 Mitt.: Das Verhalten einiger ortho-hydroxyheterochalcone under dem einvirkung von hydrazinen. 
FARMACIA, 2020, Vol. 68, 3

Arch Pharm (Weinheim), 1992; 325: 613-615, (available in German).

32. Marc G, Stana A, Pîrnău A, Vlase L, Vodnar DC, Duma M, Tiperciuc B, Oniga O, 3,5-Disubstituted Thiazolidine-2,4-Diones: Design, Microwave-Assisted Synthesis, Antifungal Activity, and ADMET Screening. SLAS Discov: Adv Sci Drug Discov., 2018; 23(8): 807-814.

33. Mbaveng AT, Kuete V, Efferth T, Potential of Central, Eastern and Western Africa medicinal plants for cancer therapy: spotlight on resistant cells and molecular targets. Front Pharmacol., 2017; 8: 343: $1-31$.

34. Morris GM, Huey R, Lindstrom W, Sanner MF, Belew RK, Goodsell DS, Olson AJ, AutoDock4 and Auto DockTools4: Automated Docking with Selective Receptor Flexibility. J Comput Chem., 2009; 30(16): 2785-2791.

35. O'Brien J, Wilson I, Orton T, Pognan F, Investigation of the Alamar Blue (resazurin) fluorescent dye for the assessment of mammalian cell cytotoxicity. Eur J Biochem., 2000; 267(17): 5421-5426.

36. Pettersen EF, Goddard TD, Huang CC, Couch GS, Greenblatt DM, Meng EC, Ferrin TE, UCSF Chimera - a visualization system for exploratory research and analysis. J Comput Chem., 2004; 25: 1605-1612.

37. Ragab FA, Yahya TA, El-Naa MM, Arafa RK, Design, synthesis and structure-activity relationship of novel semi-synthetic flavonoids as antiproliferative agents. Eur J Med Chem., 2014; 82: 506-520.

38. Sievers F, Wilm A, Dineen D, Gibson TJ, Karplus K, Li W, Lopez R, McWilliam H, Remmert M, Söding J, Thompson JD, Higgins DG, Fast, scalable generation of high-quality protein multiple sequence alignments using Clustal Omega. Mol Syst Biol., 2011; 7: 539: $1-6$.

39. Simiti I, Zaharia V, Mager S, Horn M, KötelesPopa T, Heterocyclen 67 Mitt.: Darstellung und charakterisierung einiger 2-(2-aryl-thiazol-4-yl)-3- hydroxy-chromone. Arch Pharm (Weinheim), 1991; 324: 913-915, (available in German).

40. Staker BL, Feese MD, Cushman M, Pommier Y, Zembower D, Stewart L, Burgin AB, Structures of three classes of anticancer agents bound to the human topoisomerase I-DNA covalent complex. $J$ Med Chem., 2005; 48(7): 2336-2345.

41. Stana A, Vodnar DC, Marc G, Benedec D, Tiperciuc B, Tamaian R, Oniga O, Antioxidant activity and antibacterial evaluation of new thiazolin-4-one derivatives as potential tryptophanyl-tRNA synthetase inhibitors. J Enzyme Inhib Med Chem., 2019; 34(1): 898-908.

42. Turan-Zitouni G, Altıntop MD, Özdemir A, Kaplancıklı ZA, Çiftçi GA, Temel HE, Synthesis and evaluation of bis-thiazole derivatives as new anticancer agents. Eur J Med Chem., 2016; 107(Supplement C): 288294.

43. Wu CC, Li YC, Wang YR, Li TK, Chan NL, On the structural basis and design guidelines for type II topoisomerase-targeting anticancer drugs. $\mathrm{Nucl}$ Acids Res., 2013; 41(22): 10630-10640.

44. Xie Y, Kril LM, Yu T, Zhang W, Frasinyuk MS, Bondarenko SP, Kondratyuk KM, Hausman E, Martin ZM, Wyrebek PP, Liu X, Deaciuc A, Dwoskin LP, Chen J, Zhu H, Zhan CG, Sviripa VM, Blackburn J, Watt DS, Liu C, Semisynthetic aurones inhibit tubulin polymerization at the colchicine-binding site and repress PC-3 tumour xenografts in nude mice and myc-induced T-ALL in zebrafish. Sci Rep., 2019; 9(1): 6439: 1-15.

45. Zaharia V, Imre S, Matinca D, Chirtoc I, Fagarasan E, Heterocycles XVII. Synthesis and characterisation of some $\alpha, \beta$-unsaturated carbonyls with thiazolo[3,2-b] [1,2,4]triazole structure. Clujul Med., 2002; 1: 99-104.

46. Zhang N, Yang J, Li K, Luo J, Yang S, Song JR, Chen C, Pan WD, Synthesis of Flavone Derivatives via $\mathrm{N}$-Amination and Evaluation of Their Anticancer Activities. Molecules, 2019; 24(15): 2723: 1-10. 